\title{
An Air-Stable Dimeric Ru-S Complex with an NHC as Ancillary Ligand for Cooperative Si-H Bond Activation
}

\author{
Susanne Bähr, Antoine Simonneau, Elisabeth Irran, and Martin Oestreich* \\ Institut für Chemie, Technische Universität Berlin, \\ Strasse des 17. Juni 115, D-10623 Berlin, Germany \\ martin.oestreich@tu-berlin.de
}

\section{Supporting Information}

Table of Contents

1 General Information

2 Preparation and Characterization Data for Ruthenium(II) Complexes 7 and S4 4

2.1 Preparation and Analytical Data for Ruthenium NHC Chloride Complex $7 \quad$ S4

2.2 Preparation and Analytical Data for Dimeric Ruthenium NHC Complex $4 \quad$ S6

3 Ruthenium NHC Complexes as Catalysts for Dehydrogenative Silylation S8 and Hydrosilylation Reactions

3.1 Ethyldimethyl[(1-phenylvinyl)oxy]silane (10) and Ethyldimethyl(1-phenylethoxy)silane (11)

3.1.1 Synthesis of $\mathbf{1 0}$ and $\mathbf{1 1}$ Using Dimeric Ruthenium NHC Complex $\mathbf{4}$

3.1.2 Synthesis of $\mathbf{1 0}$ and $\mathbf{1 1}$ Using Ruthenium NHC Chloride Complex $\mathbf{7}$ and $\mathrm{NaBAr}_{4}$

3.1.3 NMR Spectroscopic Data for $\mathbf{1 0}$ and $\mathbf{1 1}$

3.2 3-Dimethylphenylsilyl-1-methyl-1H-indole (13)

3.2.1 Synthesis of $\mathbf{1 3}$ Using Dimeric Ruthenium NHC Complex 4

3.2.2 Synthesis of $\mathbf{1 3}$ Using Ruthenium NHC Chloride Complex $\mathbf{7}$ and $\mathrm{NaBAr}_{4}$

3.2.3 NMR Spectroscopic Data for $\mathbf{1 3}$

3.3 5-Methyl-5-phenyl-5H-dibenzo[b, $d]$ silole (15)

3.3.1 Synthesis of 15 Using Dimeric Ruthenium NHC Complex 4

3.3.2 Synthesis of $\mathbf{1 5}$ Using Ruthenium NHC Chloride Complex $\mathbf{7}$ and $\mathrm{NaBAr}_{4}$ 
3.3.3 NMR Spectroscopic Data for 15

3.4 Triethylsilyl formate (16), Bis(triethylsilyl)acetal (17), and Triethyl(methoxy)- $\quad$ S11 silane (18)

3.4.1 Synthesis of $\mathbf{1 6}$ and $\mathbf{1 7}$ Using Dimeric Ruthenium NHC Complex 4

3.4.2 Synthesis of 16-18 Using Ruthenium NHC Chloride Complex $\mathbf{7} \quad$ S11 and $\mathrm{NaBAr}_{4}$

3.4.3 NMR Spectroscopic Data for 16-18 S12

3.5 1-Dimethylphenylsilyl-1,4-dihydropyridine (20) S12

3.5.1 Synthesis of 20 Using Dimeric Ruthenium NHC Complex $4 \quad$ S12

3.5.2 Synthesis of 20 Using Ruthenium NHC Chloride Complex 7 and $\operatorname{NaBAr}_{4} \quad \mathrm{~S} 12$

3.5.3 NMR Spectroscopic Data for $20 \quad$ S12

4 Mechanistic Experiments $\mathrm{S} 13$

4.1 Partial Formation of Monomeric Ruthenium NHC Complex 8 at Elevated S13 Temperatures

4.2 Partial Formation of Pyridine-Coordinated Monomeric Complex 8·py S14

$5 \quad$ NMR Spectra $\quad$ S17

6 References $\quad$ S39 


\section{$1 \quad$ General Information}

All reactions were performed in flame-dried glassware using an MBraun glove box $\left(\mathrm{O}_{2}<10\right.$ ppm, $\mathrm{H}_{2} \mathrm{O}<1 \mathrm{ppm}$ ) or conventional Schlenk techniques under a static pressure of argon (glove box) or nitrogen. Microwave reactions were performed in a "Discover-SP" microwave by CEM using $35 \mathrm{~mL}$ microwave flasks and caps made of silicone ("ActiVent"). All microwave reactions were performed in "open-vessel mode" allowing perforation of the cap with a cannula ( $0.9 \mathrm{~mm}$ thickness) to liberate the formed dihydrogen gas. Heating of microwave reactions was performed in "dynamic mode" and mixtures were stirred with "high mixing". For reactions with $\mathrm{CO}_{2}$, Norell ${ }^{\circledR}$ Intermediate Pressure Valved NMR tubes with a volume of $1.4 \mathrm{~mL}$ were used. These reactions were run with $0.4 \mathrm{~mL}$ solvent leaving ca. $1.0 \mathrm{~mL}$ of head space. The $\mathrm{CO}_{2}$ stoichiometries were approximated with ideal gas law at $25^{\circ} \mathrm{C}$ (ca. $2.0 \mathrm{mmol}$ $\mathrm{CO}_{2}$ ). Liquids and solutions were transferred with syringes. $\mathrm{CH}_{2} \mathrm{Cl}_{2}$, benzene, and $n$-hexane were purified and dried using a MBraun solvent system. Toluene was distilled over sodium, degassed, and stored in the glove box over $4 \AA$ molecular sieves. $\mathrm{CD}_{2} \mathrm{Cl}_{2}$ (purchased from Eurisotop), $\mathrm{C}_{6} \mathrm{D}_{6}$ (from Sigma Aldrich) and $0-\mathrm{Cl}_{2} \mathrm{C}_{6} \mathrm{D}_{4}$ (from Deutero $\mathrm{GmbH}$ ) were degassed and stored in a glove box over $4 \AA$ molecular sieves. $\mathrm{CDCl}_{3}$ (purchased from Eurisotop) was stored over $\mathrm{Cs}_{2} \mathrm{CO}_{3}$. Technical grade solvents for extraction and chromatography (cyclohexane, $\mathrm{CH}_{2} \mathrm{Cl}_{2}$, ethyl acetate, $n$-pentane, and tert-butylmethyl ether) were distilled prior to use. Carbene $5,{ }^{[\mathrm{S} 1]}$ ruthenium complex $6{ }^{[\mathrm{S} 2]}{ }^{\mathrm{NaBAr}}{ }_{4},{ }^{[\mathrm{S} 3]}$ and $1,1^{\prime}$-biphenyl-2-ylmethylphenylsilane $(\mathbf{1 4})^{[\mathrm{S4}]}$ were synthesized according to reported procedures. Triethylsilane (purchased from Sigma Aldrich) and ethyldimethylsilane (purchased from Sigma Aldrich), 1methylindole (12, purchased from abcr) and pyridine (19, purchased from Alfa Aesar) were degassed and stored in a glove box over $4 \AA$ molecular sieves. Dimethylphenylsilane (purchased from abcr) and acetophenone (9) were distilled, degassed and stored in a glove box over $4 \AA$ molecular sieves. Carbon dioxide was obtained from Air Liquide $\left[\mathrm{CO}_{2} \mathrm{~N} 35\left(\mathrm{CO}_{2}\right.\right.$ $\geq 99.95 \mathrm{vol} \%, \mathrm{H}_{2} \mathrm{O}<100 \mathrm{ppmv}, \mathrm{O}_{2}<10 \mathrm{ppmv}$ )]. All other commercially available reagents were used as received. Analytical thin-layer chromatography (TLC) was performed on silica gel SIL G-25 glass plates from Macherey-Nagel. Flash column chromatography was performed on silica gel 60 (40-63 $\mu \mathrm{m}, 230-400$ mesh, ASTM) by Merck using the indicated solvents. ${ }^{1} \mathrm{H},{ }^{11} \mathrm{~B},{ }^{13} \mathrm{C},{ }^{19} \mathrm{~F}$, and ${ }^{29} \mathrm{Si} N \mathrm{NM}$ spectra were recorded in $\mathrm{CDCl}_{3}, \mathrm{C}_{6} \mathrm{D}_{6}, \mathrm{CD}_{2} \mathrm{Cl}_{2}$ or o$\mathrm{Cl}_{2} \mathrm{C}_{6} \mathrm{D}_{4}$ on Bruker AV 400, Bruker AV 500 and Bruker AV 700 instruments. Chemical shifts are reported in parts per million (ppm) downfield from tetramethylsilane and are referenced to the residual solvent resonance as the internal standard $\left(\mathrm{CHCl}_{3}: \delta=7.26 \mathrm{ppm}\right.$ for ${ }^{1} \mathrm{H} \mathrm{NMR}$ and $\mathrm{CDCl}_{3}: \delta=77.16$ ppm for ${ }^{13} \mathrm{C} N M R, \mathrm{C}_{6} \mathrm{D}_{5} \mathrm{H}: \delta=7.16 \mathrm{ppm}$ for ${ }^{1} \mathrm{H}$ NMR and $\mathrm{C}_{6} \mathrm{D}_{6}: \delta=$ 128.06 ppm for ${ }^{13} \mathrm{C} \mathrm{NMR}, \mathrm{CHDCl}_{2}: \delta=5.32 \mathrm{ppm}$ for ${ }^{1} \mathrm{H} \mathrm{NMR}$ and $\mathrm{CD}_{2} \mathrm{Cl}_{2}: \delta=53.84 \mathrm{ppm}$ for ${ }^{13} \mathrm{C} N M R, O-\mathrm{Cl}_{2} \mathrm{C}_{6} \mathrm{D}_{4}: \delta=6.94$ and 7.20 for $\left.{ }^{1} \mathrm{H} \mathrm{NMR}\right) .{ }^{11} \mathrm{~B},{ }^{19} \mathrm{~F}$, and ${ }^{29} \mathrm{Si}$ NMR spectra were calibrated according to the IUPAC recommendation using a unified chemical shift scale based on the proton resonance of trimethylsilane as primary reference. Data are reported as follows: chemical shift, multiplicity $\left(s_{b r}=\right.$ broad singlet, $s=$ singlet, $d=$ doublet, $t=$ triplet, $q=$ quartet, $\mathrm{m}=$ multiplet), coupling constant $(\mathrm{Hz})$, integration, and, if necessary, assignment. High-resolution mass spectrometry (HRMS) and elemental analysis were performed by the Analytical Facility of the Institut für Chemie, Technische Universität Berlin. Data for the single crystal structure determination were collected with an Agilent SuperNova diffractometer equipped with a CCD area Atlas detector and a mirror monochromator by utilizing $\mathrm{Cu}-\mathrm{Ka}$ radiation $(\lambda=1.5418 \AA$ ). Software packages used: CrysAlis PRO for data collection, cell refinement, and data reduction, ${ }^{[S 5]}$ SHELXS-97 for structure solution, ${ }^{[\mathrm{S} 6]}$ SHELXL-97 for structure refinement ${ }^{[\mathrm{S7]}]}$ and Mercury $3.1 .1^{[\mathrm{S} 8]}$ for graphics. 


\section{Preparation and Characterization Data for Ruthenium(II) Complexes 7 and 4}

\subsection{Preparation and Analytical Data for Ruthenium NHC Chloride Complex 7}

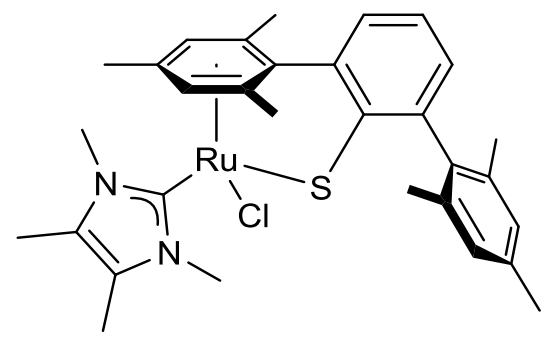

$$
\begin{gathered}
7 \\
\mathrm{C}_{31} \mathrm{H}_{37} \mathrm{CIN}_{2} \mathrm{RuS} \\
\mathrm{M}=606.23 \mathrm{~g} / \mathrm{mol}
\end{gathered}
$$

In a glove box, ruthenium complex 6 ( $246 \mathrm{mg}, 399$ umol, 1.00 equiv) and carbene 5 (50 mg, $0.40 \mathrm{mmol}, 1.0$ equiv) were weighed into a $25 \mathrm{~mL}$ Schlenk flask and toluene $(5 \mathrm{~mL})$ was added. The flask was equipped with a septum, removed from the glove box, and connected to the Schlenk line under nitrogen atmosphere. The mixture was stirred at $90{ }^{\circ} \mathrm{C}$ for $3 \mathrm{~d}$ and then diluted with $\mathrm{CH}_{2} \mathrm{Cl}_{2}$ (ca. $10 \mathrm{~mL}$ ). The volatiles were removed under reduced pressure, and the resulting brown solid was suspended in cyclohexane (ca. $5 \mathrm{~mL}$ ). The mixture was filtered, and the solid was washed with cyclohexane $(3 \times 10 \mathrm{~mL})$ and benzene $(1 \times 3 \mathrm{~mL})$. Drying in high vacuum afforded ruthenium NHC chloride complex 7 (216 mg, 89\%) as a brown solid. Single crystals of 7 suitable for $\mathrm{X}$-ray diffraction were obtained by slow vaporization of a $\mathrm{CH}_{2} \mathrm{Cl}_{2} / n$-pentane (approx. 20:1) solution. ${ }^{1} \mathbf{H} \mathbf{N M R}\left(400 \mathrm{MHz}, \mathrm{CD}_{2} \mathrm{Cl}_{2}\right)$ : $\delta / p p m=1.79(\mathrm{~s}, 3 \mathrm{H}), 1.99(\mathrm{~s}, 3 \mathrm{H}), 2.01(\mathrm{~s}, 3 \mathrm{H}), 2.07(\mathrm{~s}$ r, 6H), $2.13(\mathrm{~s}, 3 \mathrm{H}), 2.16(\mathrm{~s}, 3 \mathrm{H}), 2.30$ $(\mathrm{s}, 3 \mathrm{H}), 3.47\left(\mathrm{~s}_{\mathrm{br}}, 3 \mathrm{H}\right), 3.82\left(\mathrm{~s}_{\mathrm{br}}, 3 \mathrm{H}\right), 4.82(\mathrm{~s}, 1 \mathrm{H}), 5.44(\mathrm{~s}, 1 \mathrm{H}), 6.88(\mathrm{dd}, J=7.4,1.4 \mathrm{~Hz}, 1 \mathrm{H})$, $6.91\left(\mathrm{~s}_{\mathrm{br}}, 1 \mathrm{H}\right), 6.92\left(\mathrm{~s}_{\mathrm{br}}, 1 \mathrm{H}\right), 7.01(\mathrm{t}, J=7.4 \mathrm{~Hz}, 1 \mathrm{H}), 7.08(\mathrm{dd}, J=7.4,1.4 \mathrm{~Hz}, 1 \mathrm{H}) .{ }^{13} \mathrm{C}\left\{{ }^{1} \mathrm{H}\right\}$ NMR (126 MHz, $\left.\mathrm{CD}_{2} \mathrm{Cl}_{2}\right): \delta / p p m=9.7(\mathrm{~s}), 16.9\left(\mathrm{~s}_{\mathrm{br}}\right), 17.8\left(\mathrm{~s}_{\mathrm{br}}, 2 \mathrm{C}\right), 18.7(\mathrm{~s}), 20.6(\mathrm{~s}), 20.7(\mathrm{~s})$, 21.2 (s), 36.7 ( $\left.\mathrm{s}_{\mathrm{br}}, 2 \mathrm{C}\right), 81.5$ (s), 83.1 (s), 93.5 (s), 94.2 (s), 97.2 (s), 106.5 (s), 121.2 (s), 126.6 ( $\left.\mathrm{s}_{\mathrm{br}}, 2 \mathrm{C}\right), 127.0$ (s), 128.0 (s), 128.3 (s), 128.9 (s), 135.6 (s), 136.4 (s), 137.3 (s), 137.7 (s), 138.5 (s), 141.8 (s), 160.6 (s), 172.3 (s). HRMS (ESI) calculated for $\mathrm{C}_{31} \mathrm{H}_{37} \mathrm{~N}_{2}$ RuS $^{+}$[(M$\left.\mathrm{Cl}^{-}\right)^{+}$]: 571.1715; found: 571.1709. EA calculated for $\mathrm{C}_{31} \mathrm{H}_{37} \mathrm{CIN}_{2} \mathrm{RuS}$ : C, $61.42 ; \mathrm{H}, 6.15 ; \mathrm{N}$, 4.62; found: $\mathrm{C}, 61.53 ; \mathrm{H}, 6.20 ; \mathrm{N}, 4.57$. Molecular structure and X-ray data: CCDC1448587 contains the supplementary crystallographic data for this compound. These data can be obtained free of charge at www.ccdc.cam.ac.uk/conts/retrieving.html [or from the Cambridge Crystallographic Data Centre, 12 Union Road, Cambridge CB2 1EZ, UK; fax: (internat.) +44(1223)336-033, E-mail: deposit@ccdc.cam.ac.uk]. 


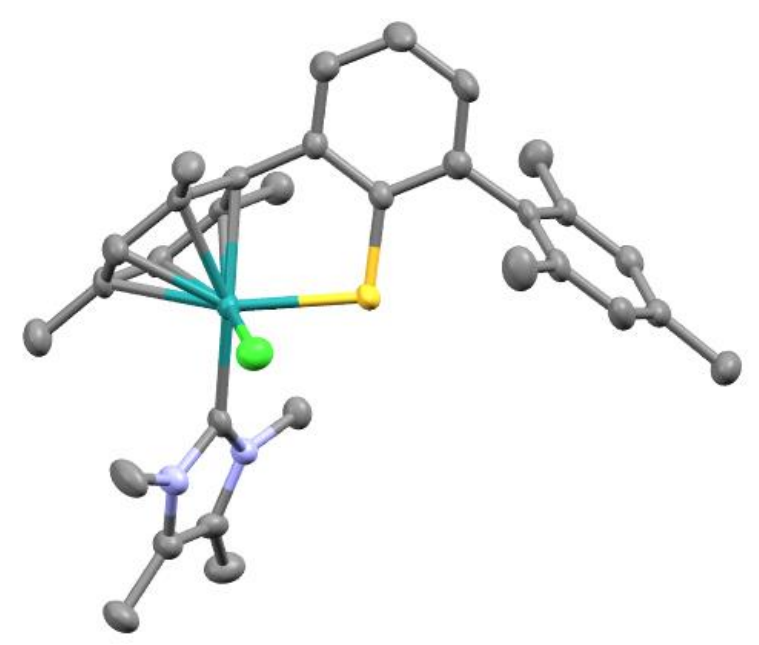

Figure S1. Molecular structure of $\mathbf{7}$. Thermal ellipsoids represent $50 \%$ probability level. $\mathrm{H}$ atoms are removed for clarity.

Empirical formula
Formula weight
Temperature
Wavelength
Crystal system
Space group
Unit cell dimensions

Volume

Z

Density (calculated)

Absorption coefficient

$\mathrm{F}(000)$

Crystal size

Theta range for data collection

Index ranges

Reflections collected

Independent reflections

Completeness to theta $=67.49^{\circ}$

Absorption correction

Max. and min. transmission

Refinement method

Data / restraints / parameters

Goodness-of-fit on $\mathrm{F}^{2}$

Final $R$ indices [l>2sigma(I)]

$R$ indices (all data)

Largest diff. peak and hole
$\mathrm{C}_{31} \mathrm{H}_{37} \mathrm{ClN}_{2} \mathrm{RuS}(7)$

606.21

$150.00(10) \mathrm{K}$

$1.54184 \AA$

Monoclinic

$\mathrm{P} 21 / \mathrm{n}$

$a=7.49867(19) \AA$

$\mathrm{b}=23.3134(5) \AA$

$c=16.1586(4) \AA$

$\alpha=90^{\circ}$

$\beta=93.545(2)^{\circ}$

$Y=90^{\circ}$

2819.43(11) $\AA^{3}$

4

$1.428 \mathrm{Mg} / \mathrm{m}^{3}$

$6.224 \mathrm{~mm}^{-1}$

1256

$0.31 \times 0.03 \times 0.02 \mathrm{~mm}^{3}$

3.33 to $67.49^{\circ}$

$-8<=\mathrm{h}<=8,-27<=\mathrm{k}<=18,-19<=\mathrm{|}<=19$

10426

$5063[\mathrm{R}$ (int) $=0.0643]$

$100.0 \%$

Semi-empirical from equivalents

0.8856 and 0.2485

Full-matrix least-squares on $\mathrm{F}^{2}$

5063 / 0 / 335

1.009

$\mathrm{R} 1=0.0464, \mathrm{wR} 2=0.0929$

$\mathrm{R} 1=0.0756, \mathrm{wR} 2=0.1073$

0.693 and -0.834 e. $\AA^{-3}$ 


\subsection{Preparation and Analytical Data for Dimeric Ruthenium NHC Complex 4}

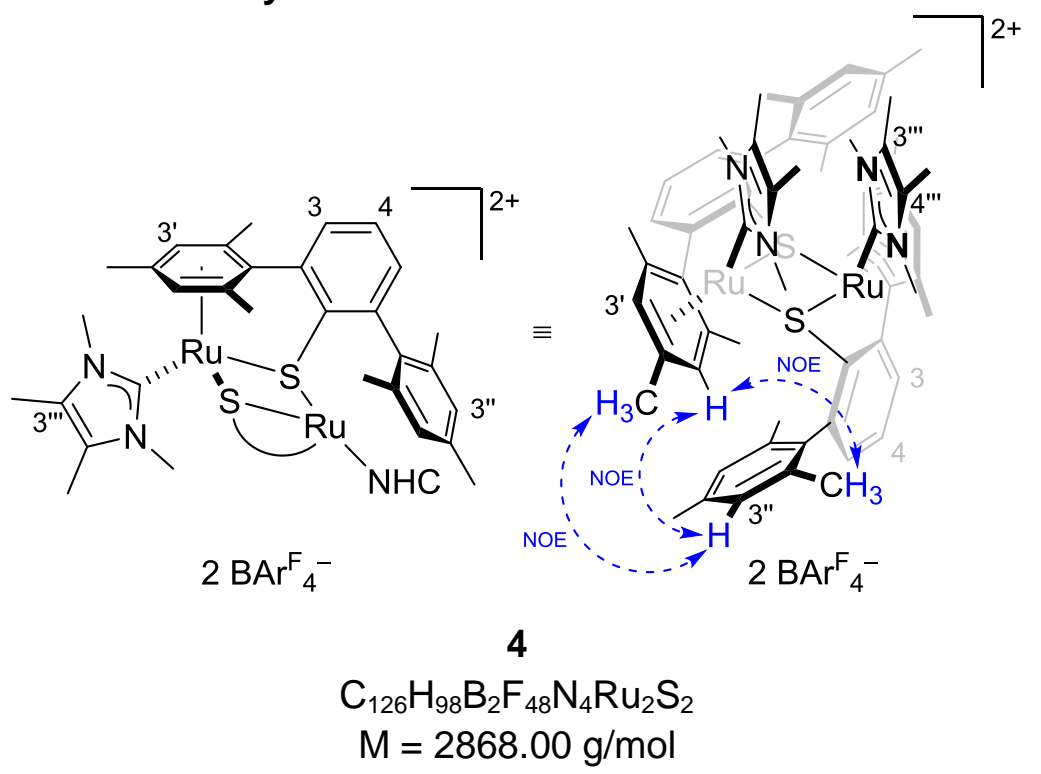

In a glove box, ruthenium chloride 7 (33 mg, $54 \mu \mathrm{mol}, 1.0$ equiv) and $\mathrm{NaBAr}_{4}$ (48 mg, $54 \mu \mathrm{mol}, 1.0$ equiv) were weighed into a $5 \mathrm{~mL}$ glass vial and $\mathrm{CH}_{2} \mathrm{Cl}_{2}(2 \mathrm{~mL})$ was added. The solution was stirred for $15 \mathrm{~min}$ at room temperature during which the initial immediate color change from red to dark blue was followed by another color change to brown. The vial was removed from the glove box and the solution diluted with $\mathrm{CH}_{2} \mathrm{Cl}_{2}(5 \mathrm{~mL})$. The mixture was filtered over a PTFE syringe filter, and the solvent of the filtrate was removed under reduced pressure. The resulting solid was washed with benzene $(3 \mathrm{~mL})$. Drying in high vacuum afforded dimeric ruthenium NHC complex 4 (77 mg, 99\%) as a brown solid. Single crystals of 4 suitable for $X$-ray diffraction were obtained by slow vaporization of a $\mathrm{CH}_{2} \mathrm{Cl}_{2}$ solution.

${ }^{1} \mathrm{H}$ NMR $\left(400 \mathrm{MHz}, \mathrm{CD}_{2} \mathrm{Cl}_{2}\right): \delta / p p m=1.19\left(\mathrm{~s}, 6 \mathrm{H}, 2\right.$ '- $\left.\mathrm{CH}_{3}\right), 1.26\left(\mathrm{~s}, 6 \mathrm{H}, 4{ }^{\prime}-\mathrm{CH}_{3}\right), 1.86(\mathrm{~s}, 6 \mathrm{H}$,

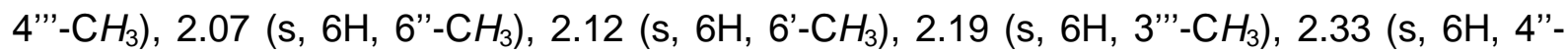

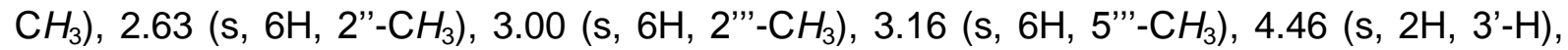
$5.53\left(\mathrm{~s}, 2 \mathrm{H}, 5\right.$ '-H), $6.92\left(\mathrm{~s}_{\mathrm{br}}, 2 \mathrm{H}, 5\right.$ "'-H), $7.20\left(\mathrm{~s}_{\mathrm{br}}, 2 \mathrm{H}, 3\right.$ "'-H), $7.35\left(\mathrm{dd},{ }^{3} J_{\mathrm{H}, \mathrm{H}}=7.6 \mathrm{~Hz},{ }^{4} J_{\mathrm{H}, \mathrm{H}}=\right.$ $1.5 \mathrm{~Hz}, 2 \mathrm{H}, 5-\mathrm{H}), 7.43\left(\mathrm{dd},{ }^{3} \mathrm{~J}_{\mathrm{H}, \mathrm{H}}=7.5 \mathrm{~Hz},{ }^{4} \mathrm{~J}_{\mathrm{H}, \mathrm{H}}=1.6 \mathrm{~Hz}, 2 \mathrm{H}, 3-\mathrm{H}\right), 7.54-7.57(\mathrm{~m}, 8 \mathrm{H}, p-\mathrm{CH}-$ $\left.\mathrm{BAr}^{\mathrm{F}}\right), 7.62\left(\mathrm{t},{ }^{3} \mathrm{~J}_{\mathrm{H}, \mathrm{H}}=7.5 \mathrm{~Hz}, 2 \mathrm{H}, 4-\mathrm{H}\right), 7.70-7.74\left(\mathrm{~m}, 16 \mathrm{H}, m-\mathrm{CH}-\mathrm{BAr}^{\mathrm{F}}\right) .{ }^{13} \mathrm{C}\left\{{ }^{1} \mathrm{H}\right\} \mathrm{NMR}$ $\left(101 \mathrm{MHz}, \mathrm{CD}_{2} \mathrm{Cl}_{2}\right): \delta / p p m=9.2\left(\mathrm{~s}, 4\right.$ '”- $\left.-\mathrm{CH}_{3}\right), 10.0$ (s, 3'”- $\left.-\mathrm{CH}_{3}\right), 17.4$ (s, 6'- $\left.\mathrm{CH}_{3}\right), 18.0$ (2s, 2'$\mathrm{CH}_{3}, 4$ '- $\mathrm{CH}_{3}$ ), 20.9 (s, 4"- $\mathrm{CH}_{3}$ ), 23.1, 23.2 (2s, 2" $-\mathrm{CH}_{3}, 6$ "'- $\mathrm{CH}_{3}$ ), 36.1 (s, 5'"- $-\mathrm{CH}_{3}$ ), 37.8 (s, 2'"$\mathrm{CH}_{3}$ ), 85.9 (s, C-5'), 88.0 (s, C-3'), 90.7 (s, C-2'), 94.2 (s, C-4'), 97.5 (s, C-6'), 111.1 (s, C-1'), $117.9\left(\mathrm{~s}_{\mathrm{br}}, \mathrm{p}-\mathrm{CH}-\mathrm{BAr}_{4}\right), 124.9\left(\mathrm{q},{ }^{1} \mathrm{~J}_{\mathrm{C}, \mathrm{F}}=272 \mathrm{~Hz}, \mathrm{CF}_{3}\right), 127.8$ (s, C-3"'), 128.8 (s, C-3), 129.0 (s, C-4), 129.1 (s, C-4"'), 129.1-129.7 (m, m- $C_{\text {q }}-$ BAr $_{4}$ ), 130.0 (s, C-5"), 130.9 (s, C-3"), 135.2 ( $\mathrm{s}_{\mathrm{br}}, \mathrm{o}-\mathrm{CH}-\mathrm{BAr}_{4}{ }_{4}$ ), 135.9 (s, C-2"), 136.1 (s, C-5), 137.2 (s, C-1"), 137.5 (s, C-6"), 139.4 (s, C-4"), 142.8 (s, C-2 or C-6), 145.3 (s, C-1), 146.0 (s, C-2 or C-6), 162.1 (q, ${ }^{1} J_{C, B}=$ $\left.49.8 \mathrm{~Hz}, i-\mathrm{C}_{\mathrm{q}}-\mathrm{BAr}^{\mathrm{F}}{ }_{4}\right), 167.0$ (s, C1 $\left.{ }^{\prime \prime \prime}\right) .{ }^{11} \mathrm{~B}\left\{{ }^{1} \mathrm{H}\right\}$ NMR $\left(160 \mathrm{MHz}, \mathrm{CD}_{2} \mathrm{Cl}_{2}\right): \delta / p p m=-6.6 .{ }^{19} \mathrm{~F}\left\{{ }^{1} \mathrm{H}\right\}$ NMR $\left(471 \mathrm{MHz}, \mathrm{CD}_{2} \mathrm{Cl}_{2}\right): \delta / p p m=-62.8$. HRMS $(\mathrm{APCl})$ calculated for $\mathrm{C}_{62} \mathrm{H}_{74} \mathrm{~N}_{4} \mathrm{Ru}_{2} \mathrm{~S}_{2}{ }^{2+}[(\mathrm{M}-$ $\left.2 \mathrm{BAr}_{4}{ }_{4}\right)^{2+}$ ]: 571.1715; found: 571.1718. EA calculated for $\mathrm{C}_{126} \mathrm{H}_{98} \mathrm{~B}_{2} \mathrm{~F}_{48} \mathrm{~N}_{4} \mathrm{Ru}_{2} \mathrm{~S}_{2}: \mathrm{C}, 52.77 ; \mathrm{H}$, 3.44; N, 1.95; S, 2.24; found: C, 50.10; H, 3.70; N, 1.94; S, 1.58 (no better values obtained as a result of the fluorine-rich nature of the compound). Molecular structure and X-ray Data: CCDC-1448586 contains the supplementary crystallographic data for this compound. These data can be obtained free of charge at www.ccdc.cam.ac.uk/conts/retrieving.html [or from the Cambridge Crystallographic Data Centre, 12 Union Road, Cambridge CB2 1EZ, UK; fax: (internat.) +44(1223)336-033, E-mail: deposit@ccdc.cam.ac.uk]. 


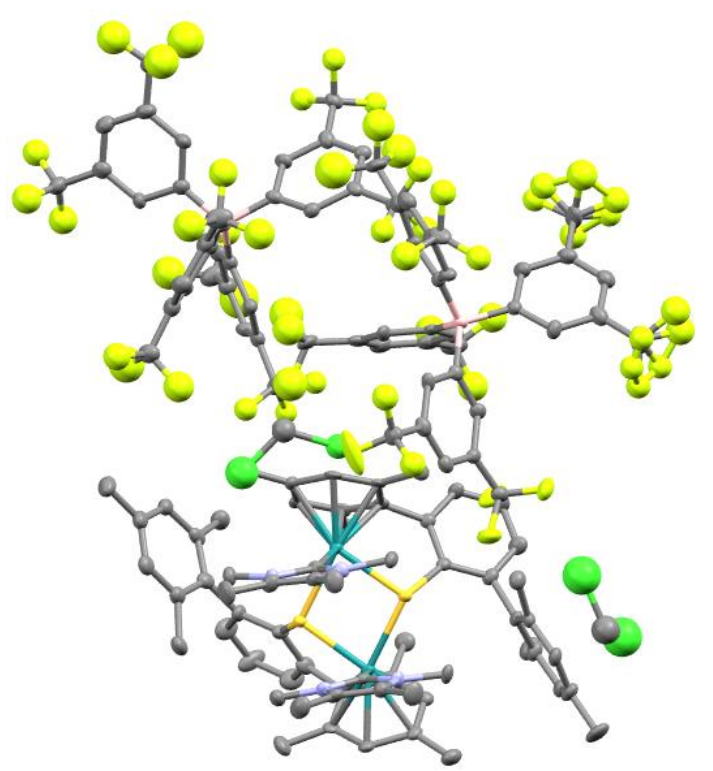

Figure S2. Molecular structure of $4 \cdot 2 \mathrm{CH}_{2} \mathrm{Cl}_{2}$. Thermal ellipsoids represent $50 \%$ probability level. $\mathrm{H}$ atoms are removed for clarity.

Empirical formula
Formula weight
Temperature
Wavelength
Crystal system
Space group
Unit cell dimensions

Volume

Z

Density (calculated)

Absorption coefficient

$\mathrm{F}(000)$

Crystal size

Theta range for data collection

Index ranges

Reflections collected

Independent reflections

Completeness to theta $=67.50^{\circ}$

Absorption correction

Max. and min. transmission

Refinement method

Data / restraints / parameters

Goodness-of-fit on $\mathrm{F}^{2}$

Final $\mathrm{R}$ indices [l>2sigma(I)]

$R$ indices (all data)

Largest diff. peak and hole
$\mathrm{C}_{128} \mathrm{H}_{102} \mathrm{~B}_{2} \mathrm{Cl}_{4} \mathrm{~F}_{48} \mathrm{~N}_{4} \mathrm{Ru}_{2} \mathrm{~S}_{2}\left(4 \cdot 2 \mathrm{CH}_{2} \mathrm{Cl}_{2}\right)$

3037.82

$150.00(10) \mathrm{K}$

$1.54184 \AA$

Monoclinic

$\mathrm{P} 21 / \mathrm{n}$

$a=24.3853(3) \AA$

$\mathrm{b}=16.0918(2) \AA$

$\mathrm{c}=33.0002(4) \AA$

$\alpha=90^{\circ}$

$\beta=102.9546(13)^{\circ}$

$\mathrm{Y}=90^{\circ}$

$12619.8(3) \AA^{3}$

4

$1.599 \mathrm{Mg} / \mathrm{m}^{3}$

$4.125 \mathrm{~mm}^{-1}$

6112

$0.26 \times 0.08 \times 0.07 \mathrm{~mm}^{3}$

2.55 to $67.50^{\circ}$

$-29<=\mathrm{h}<=20,-19<=\mathrm{k}<=17,-39<=\mathrm{l}<=34$

51689

$22713[\mathrm{R}$ (int) $=0.0651]$

$99.9 \%$

Semi-empirical from equivalents

0.7611 and 0.4135

Full-matrix-block least-squares on $\mathrm{F}^{2}$ 22713 / 0 / 1785

1.085

$\mathrm{R} 1=0.1032, \mathrm{wR} 2=0.2242$

$\mathrm{R} 1=0.1394, \mathrm{wR} 2=0.2494$

3.149 and -2.250 e. $\AA^{-3}$ 
3 Ruthenium NHC Complexes as Catalysts for Dehydrogenative Silylation and Hydrosilylation Reactions

3.1 Ethyldimethyl[(1-phenylvinyl)oxy]silane (10) and Ethyldimethyl(1-phenylethoxy)silane (11)<smiles>C=C(O[SiH2]C(C)C)c1ccccc1</smiles>

10

$\mathrm{C}_{12} \mathrm{H}_{18} \mathrm{OSi}$

$\mathrm{M}=206.36 \mathrm{~g} / \mathrm{mol}$<smiles>CC(O[SiH](C)C)c1ccccc1</smiles>

11

$\mathrm{C}_{12} \mathrm{H}_{20} \mathrm{OSi}$

$\mathrm{M}=208.38 \mathrm{~g} / \mathrm{mol}$

\subsubsection{Synthesis of 10 and 11 Using Dimeric Ruthenium NHC Complex 4}

In a glove box, ruthenium dimer $4(2.9 \mathrm{mg}, 1.0 \mu \mathrm{mol}, 0.50 \mathrm{~mol} \%)$ was weighed into a GLC vial. Acetophenone $(9,24 \mathrm{mg}, 0.20 \mathrm{mmol}, 1.0$ equiv) and ethyldimethylsilane $(88 \mathrm{mg}$, $1.0 \mathrm{mmol}, 5.0$ equiv) were added, and the resulting suspension was maintained at room temperature for $24 \mathrm{~h}$. The reaction mixture was diluted and filtered through a small pad of Celite $^{\circledR}$ layered with a small amount of silica gel using a solution of cyclohexane, tertbutylmethyl ether, and triethylamine $(80: 5: 1,0.5 \mathrm{~mL}+5 \mathrm{~mL})$. The solvents of the filtrate were removed under reduced pressure. Purification by flash chromatography on silica gel (eluent: cyclohexane/ethyl acetate 50:1) afforded a mixture of silyl enol ether $\mathbf{1 0}$ and silyl ether $\mathbf{1 1}$ (76:24, $33 \mathrm{mg}, 80 \%$ ) as a clear liquid. The ratio of $\mathbf{1 0}$ to $\mathbf{1 1}$ was determined by integration of the baseline-separated signals at 4.91 and $4.86 \mathrm{ppm}$ in the ${ }^{1} \mathrm{H}$ NMR spectrum.

\subsubsection{Synthesis of 10 and 11 Using Ruthenium NHC Chloride Complex 7 and $\operatorname{NaBAr}_{4}$}

In a glove box, ruthenium chloride $7(1.2 \mathrm{mg}, 2.0 \mu \mathrm{mol}, 1.0 \mathrm{~mol} \%)$ was weighed into a GLC vial and $\operatorname{NaBAr}_{4}(1.8 \mathrm{mg}, 2.0 \mu \mathrm{mol}, 1.0 \mathrm{~mol} \%)$ was added. Acetophenone (9, $24 \mathrm{mg}$, $0.2 \mathrm{mmol}, 1.0$ equiv) and ethyldimethylsilane ( $89 \mathrm{mg}, 1.0 \mathrm{mmol}, 5.0$ equiv) were added, and the resulting suspension was maintained at room temperature for $2 \mathrm{~h}$. The reaction mixture was diluted and filtered through a small pad of Celite ${ }^{\circledR}$ layered with a small amount of silica gel using a solution of cyclohexane, tert-butylmethyl ether and triethylamine $(80: 5: 1,0.5 \mathrm{~mL}$ $+5 \mathrm{~mL}$ ). The solvents of the filtrate were removed under reduced pressure. Purification by flash chromatography on silica gel (eluent: cyclohexane/ethyl acetate 50:1) afforded a mixture of silyl enol ether 10 and silylether $11(75: 25,39 \mathrm{mg}, 95 \%)$ as a clear liquid. The ratio of $\mathbf{1 0}$ to $\mathbf{1 1}$ was determined by integration of the baseline-separated signals at 4.91 and $4.86 \mathrm{ppm}$ in the ${ }^{1} \mathrm{H}$ NMR spectrum.

\subsubsection{NMR Spectroscopic Data for 10 and 11}

10: ${ }^{1} \mathrm{H}$ NMR $\left(500 \mathrm{MHz}, \mathrm{CDCl}_{3}\right): \delta / p p m=0.25(\mathrm{~s}, 6 \mathrm{H}), 0.75(\mathrm{q}, \mathrm{J}=7.9 \mathrm{~Hz}, 2 \mathrm{H}), 1.01$ (t, $\mathrm{J}=$ $7.9 \mathrm{~Hz}, 3 \mathrm{H}), 4.43(\mathrm{~d}, J=1.7 \mathrm{~Hz}, 1 \mathrm{H}), 4.91(\mathrm{~d}, J=1.7 \mathrm{~Hz}, 1 \mathrm{H}), 7.27-7.34(\mathrm{~m}, 3 \mathrm{H}), 7.59-7.61$ $(\mathrm{m}, 2 \mathrm{H}) .{ }^{13} \mathrm{C}\left\{{ }^{1} \mathrm{H}\right\}$ NMR $\left(126 \mathrm{MHz}, \mathrm{CDCl}_{3}\right): \delta / \mathrm{ppm}=-1.9(\mathrm{~s}, 2 \mathrm{C}), 6.8(\mathrm{~s}), 8.6(\mathrm{~s}), 91.0(\mathrm{~s})$, 125.3 (s, 2C), 128.2, 128.3 (2s, 3C), 137.7 (s), 155.9 (s). ${ }^{29} \mathrm{Si}\left\{{ }^{1} \mathrm{H}\right\}$ DEPT NMR (99 MHz, $\left.\mathrm{CDCl}_{3}\right): \delta / p p m=20.1$. 
11: ${ }^{1} \mathrm{H}$ NMR $\left(500 \mathrm{MHz}, \mathrm{CDCl}_{3}\right): \delta / \mathrm{ppm}=0.05(\mathrm{~s}, 3 \mathrm{H}), 0.06(\mathrm{~s}, 3 \mathrm{H}), 0.52-0.58(\mathrm{~m}, 2 \mathrm{H}), 0.92$ (t, $J=7.9 \mathrm{~Hz}, 3 \mathrm{H}), 1.44(\mathrm{~d}, J=6.4 \mathrm{~Hz}, 3 \mathrm{H}), 4.86(\mathrm{q}, J=6.4 \mathrm{~Hz}, 1 \mathrm{H}), 7.21-7.34(\mathrm{~m}, 1 \mathrm{H})$, 7.27-7.24 (m, 2H), 7.59-7.61 (m, 2H). ${ }^{13} \mathrm{C}\left\{{ }^{1} \mathrm{H}\right\}$ NMR $\left(126 \mathrm{MHz}, \mathrm{CDCl}_{3}\right): \delta / p p m=-2.0(\mathrm{~s})$, -2.0 (s), 6.9 (s), 8.6 (s), 27.1 (s), 70.7 (s), 125.5 (s, 2C), 126.9, 128.3 (2s, 3C), 146.8 (s). ${ }^{29} \mathrm{Si}\left\{{ }^{1} \mathrm{H}\right\}$ DEPT NMR $\left(99 \mathrm{MHz}, \mathrm{CDCl}_{3}\right): \delta / p p m=17.8$. The spectroscopic data are in accordance with those reported. ${ }^{[S 9]}$

\section{$3.2 \quad 3-D i m e t h y l p h e n y l s i l y l-1-m e t h y l-1 H$-indole (13)}<smiles>C[AsH2]c1cn(C)c2ccccc12</smiles>

13

$\mathrm{C}_{17} \mathrm{H}_{19} \mathrm{NSi}$

$\mathrm{M}=265.43 \mathrm{~g} / \mathrm{mol}$

\subsubsection{Synthesis of 13 Using Dimeric Ruthenium NHC Complex 4}

In a glove box, ruthenium dimer $4(5.6 \mathrm{mg}, 2.0 \mu \mathrm{mol}, 0.65 \mathrm{~mol} \%)$ was weighed into a GLC vial. 1-Methylindole (12, $40 \mathrm{mg}, 0.30 \mathrm{mmol}, 1.0$ equiv) and dimethylphenylsilane (55 $\mathrm{mg}$, $0.40 \mathrm{mmol}, 1.3$ equiv) were added, and the resulting suspension was maintained at $80^{\circ} \mathrm{C}$ for $41 \mathrm{~h}$. The reaction mixture was diluted with triethylamine $(0.1 \mathrm{~mL})$ and filtered through a small pad of Celite ${ }^{\circledR}$ layered with a small amount of silica gel using a solution of cyclohexane, tert-butylmethyl ether and triethylamine $(80: 5: 1,5 \mathrm{~mL})$. The solvents of the filtrate were removed under reduced pressure. The resulting liquid was dried in high vacuum for $5 \mathrm{~h}$ at $60^{\circ} \mathrm{C}$ to afford indole $13(61 \mathrm{mg}, 77 \%)$ as a clear liquid.

\subsubsection{Synthesis of 13 Using Ruthenium NHC Chloride Complex 7 and $\mathrm{NaBAr}_{4}$}

In a glove box, ruthenium chloride $7(2.4 \mathrm{mg}, 4.0 \mu \mathrm{mol}, 1.3 \mathrm{~mol} \%)$ and $\operatorname{NaBAr}_{4}(3.6 \mathrm{mg}$, $4.1 \mu \mathrm{mol}, 1.4 \mathrm{~mol} \%)$ were weighed into a GLC vial. 1-Methylindole $(\mathbf{1 2}, 40 \mathrm{mg}, 0.30 \mathrm{mmol}$, 1.0 equiv) and dimethylphenylsilane (56 mg, $0.41 \mathrm{mmol}, 1.3$ equiv) were added, and the resulting suspension was maintained at $80^{\circ} \mathrm{C}$ for $41 \mathrm{~h}$. The reaction mixture was diluted with triethylamine $(0.1 \mathrm{~mL})$ and filtered through a small pad of Celite ${ }^{\circledR}$ layered with a small amount of silica gel using a solution of cyclohexane, tert-butylmethyl ether and triethylamine (80:5:1, $5 \mathrm{~mL}$ ). The solvents of the filtrate were removed under reduced pressure. The resulting liquid was dried in high vacuum for $5 \mathrm{~h}$ at $60^{\circ} \mathrm{C}$ to afford indole 13 (48 $\left.\mathrm{mg}, 60 \%\right)$ as a clear liquid.

\subsubsection{NMR Spectroscopic Data for 13}

${ }^{1} \mathrm{H}$ NMR $\left(500 \mathrm{MHz}, \mathrm{CDCl}_{3}\right): \delta / p p m=0.61(\mathrm{~s}, 6 \mathrm{H}), 3.79(\mathrm{~s}, 3 \mathrm{H}), 7.04(\mathrm{~s}, 1 \mathrm{H}), 7.08$ (ddd, $\mathrm{J}=$ 7.9, 7.1, 0.9 Hz, 1H), 7.22-7.26 (m, 1H), 7.33-7.37 (m, 4H), 7.58 (ddd, J = 7.9, 0.9, $0.9 \mathrm{~Hz}$, $1 \mathrm{H}), 7.60-7.62(\mathrm{~m}, 2 \mathrm{H}) .{ }^{13} \mathrm{C}\left\{{ }^{1} \mathrm{H}\right\}$ NMR $\left(126 \mathrm{MHz}, \mathrm{CDCl}_{3}\right): \delta / \mathrm{ppm}=-1.2(\mathrm{~s}, 2 \mathrm{C}), 32.9(\mathrm{~s})$, 107.1 (s), 109.5 (s), 119.6 (s), 121.7 (s), 122.2 (s), 127.9, 129.0 (2s, 3C), 133.0 (s), 134.2 (s, 2C), 136.5 (s), 138.7 (s), 139.6 (s). ${ }^{29} \mathrm{Si}\left\{{ }^{1} \mathrm{H}\right\}$ DEPT NMR (99 MHz, CDCl $\left.)_{3}\right): \delta / p p m=-14.2$. The spectroscopic data are in accordance with those reported. ${ }^{[\mathrm{S} 10]}$ 


\subsection{5-Methyl-5-phenyl-5H-dibenzo[b,o]silole (15)}

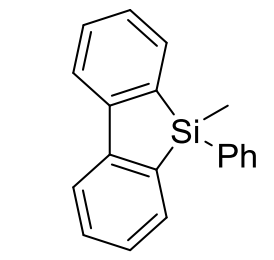

$$
\begin{gathered}
15 \\
\mathrm{C}_{19} \mathrm{H}_{16} \mathrm{Si} \\
=272.42 \mathrm{~g} / \mathrm{mol}
\end{gathered}
$$

\subsubsection{Synthesis of 15 Using Dimeric Ruthenium NHC Complex 4}

In a glove box, ruthenium dimer $4(2.9 \mathrm{mg}, 1.0 \mu \mathrm{mol}, 0.50 \mathrm{~mol} \%)$ was weighed into a $35 \mathrm{~mL}$ microwave vessel. 1,1'-Biphenyl-2-yl-methylphenylsilane (14, $54 \mathrm{mg}, 0.20 \mathrm{mmol}, 1.0$ equiv) was weighed into a GLC vial and transferred with toluene $(0.2 \mathrm{~mL})$ into the microwave vessel. The vessel was capped, and the reaction mixture was heated in a microwave $(300 \mathrm{~W})$ for $30 \mathrm{~min}$ at $140^{\circ} \mathrm{C}$. After ramping the temperature, the cap was perforated with a cannula. The mixture was diluted with cyclohexane $(3 \mathrm{~mL})$, and all volatiles were removed under reduced pressure. Purification by flash chromatography on silica gel (eluent: cyclohexane) afforded silole 15 (37.5 mg, 69\%) as a white solid.

\subsubsection{Synthesis of 15 Using Ruthenium NHC Chloride Complex 7 and $\operatorname{NaBAr}_{4}$}

In a glove box, ruthenium chloride $7(1.2 \mathrm{mg}, 2.0 \mu \mathrm{mol}, 1.0 \mathrm{~mol} \%)$ and $\operatorname{NaBAr}_{4}(1.8 \mathrm{mg}$, $2.0 \mu \mathrm{mol}, 1.0 \mathrm{~mol} \%)$ were weighed into a $35 \mathrm{~mL}$ microwave vessel. 1,1'-Biphenyl-2-ylmethylphenylsilane $(\mathbf{1 4}, 54 \mathrm{mg}, 0.20 \mathrm{mmol}, 1.0$ equiv) was weighed into a GLC vial and transferred with toluene $(0.2 \mathrm{~mL})$ into the microwave vessel. The vessel was capped and the reaction mixture was heated in a microwave $(300 \mathrm{~W})$ for $30 \mathrm{~min}$ at $140{ }^{\circ} \mathrm{C}$. After ramping the temperature, the cap was perforated with a cannula. The mixture was diluted with cyclohexane $(3 \mathrm{~mL})$ and all volatiles were removed under reduced pressure. Purification by flash chromatography on silica gel (eluent: cyclohexane) afforded silole 15 (46 mg, 84\%) as a white solid.

\subsubsection{NMR Spectroscopic Data for 15}

${ }^{1} \mathrm{H}$ NMR $\left(500 \mathrm{MHz}, \mathrm{CDCl}_{3}\right): \delta / \mathrm{ppm}=0.75(\mathrm{~s}, 3 \mathrm{H}), 7.29(\mathrm{ddd}, J=7.2,7.2,0.9 \mathrm{~Hz}, 2 \mathrm{H}), 7.31-$ $7.34(\mathrm{~m}, 2 \mathrm{H}), 7.38-7.40(\mathrm{~m}, 1 \mathrm{H}), 7.47$ (ddd, $J=7.6,7.6,1.4 \mathrm{~Hz}, 2 \mathrm{H}), 7.56-7.58(\mathrm{~m}, 2 \mathrm{H})$, 7.66 (ddd, $J=7.2,1.3,0.8 \mathrm{~Hz}, 2 \mathrm{H}), 7.87(\mathrm{~d}, J=7.7 \mathrm{~Hz}, 2 \mathrm{H}) .{ }^{13} \mathbf{C}\left\{{ }^{1} \mathrm{H}\right\}$ NMR $\left(126 \mathrm{MHz}, \mathrm{CDCl}_{3}\right)$ : $\delta / p p m=-4.9(\mathrm{~s}), 121.1(\mathrm{~s}, 2 \mathrm{C}), 127.7(\mathrm{~s}, 2 \mathrm{C}), 128.2(\mathrm{~s}, 2 \mathrm{C}), 130.0(\mathrm{~s}), 130.6$ (s, 2C), 133.5 (s, 2C), 134.6 (s, 2C), 134.8 (s), 137.5 (s, 2C), 148.5 (s, 2C). ${ }^{29} \mathrm{Si}\left\{{ }^{1} \mathrm{H}\right\}$ DEPT NMR (99 MHz, $\left.\mathrm{CDCl}_{3}\right): \delta / p p m=-5.6$. The spectroscopic data are in accordance with those reported. ${ }^{[\mathrm{S} 4]}$ 


\section{$3.4 \quad$ Triethylsilyl formate Triethyl(methoxy)silane (18)}<smiles>CCOC=O</smiles>

16<smiles>CCOCCO</smiles>

17

$$
\mathrm{C}_{13} \mathrm{H}_{32} \mathrm{O}_{2} \mathrm{Si}_{2}
$$<smiles>CCOC</smiles>

18

$$
\mathrm{M}=160.29 \mathrm{~g} / \mathrm{mol} \quad \mathrm{M}=276.57 \mathrm{~g} / \mathrm{mol} \quad \mathrm{M}=146.31 \mathrm{~g} / \mathrm{mol}
$$

\subsubsection{Synthesis of 16 and 17 Using Dimeric Ruthenium NHC Complex 4}

In a glove box, ruthenium dimer 4 (4.7 mg, $1.6 \mu \mathrm{mol}, 2.0 \mathrm{~mol} \%)$ was weighed into a GLC vial and triethylsilane $(9.4 \mathrm{mg}, 0.081 \mathrm{mmol}, 1.0$ equiv), toluene (ca. $8 \mathrm{mg})$ and $\mathrm{C}_{6} \mathrm{D}_{6}(0.4 \mathrm{~mL})$ were added. The mixture was transferred into a medium walled valved NMR tube, the tube was sealed and a ${ }^{1} \mathrm{H}$ NMR spectrum was measured to obtain an accurate calibration of the internal standard. The tube was pressurized with carbon dioxide (>99.95 vol \%, 5.0 bar, ca. $2.0 \mathrm{mmol}$, ca. 25 equiv) and heated to $80{ }^{\circ} \mathrm{C}$. The reaction was monitored with ${ }^{1} \mathrm{H}$ NMR spectroscopy.

Table S1. Monitoring the hydrosilylation of $\mathrm{CO}_{2}$ with ruthenium dimer 4 as precatalyst.

\begin{tabular}{ccccc} 
Time & Conversion & \multicolumn{3}{c}{ Ratio } \\
\cline { 3 - 5 }$(\mathrm{h})$ & $(\%)$ & $\mathbf{1 6}$ & $\mathbf{1 7}$ & $\mathbf{1 8}$ \\
\hline 1 & 15 & 29 & 71 & $<1$ \\
3 & 40 & 22 & 88 & $<1$ \\
8 & 83 & 15 & 85 & $<1$ \\
24 & $>99$ & 17 & 83 & $<1$ \\
\hline
\end{tabular}

\subsubsection{Synthesis of 16-18 Using Ruthenium NHC Chloride Complex 7 and $\mathrm{NaBAr}_{4}$}

In a glove box, ruthenium chloride $7(2.0 \mathrm{mg}, 3.3 \mu \mathrm{mol}, 4.1 \mathrm{~mol} \%)$ and $\operatorname{NaBAr}_{4}(3.0 \mathrm{mg}$, $3.4 \mu \mathrm{mol}, 4.0 \mathrm{~mol} \%)$ were weighed into a GLC vial and triethylsilane $(9.3 \mathrm{mg}, 0.080 \mathrm{mmol}$, 1.0 equiv), toluene (ca. $8 \mathrm{mg}$ ) and $\mathrm{C}_{6} \mathrm{D}_{6}(0.4 \mathrm{~mL}$ ) were added. The mixture was transferred in to a medium walled valved NMR tube, the tube was sealed and a ${ }^{1} \mathrm{H}$ NMR spectrum was measured to obtain an accurate calibration of the internal standard. The tube was pressurized with carbon dioxide (>99.95 vol \%, 5.0 bar, ca. $2.0 \mathrm{mmol}$, ca. 25 equiv) and heated to $80^{\circ} \mathrm{C}$. The reaction was monitored with ${ }^{1} \mathrm{H}$ NMR spectroscopy.

Table S2. Monitoring the hydrosilylation of $\mathrm{CO}_{2}$ with in-situ prepared catalyst 8 .

\begin{tabular}{ccccc} 
& Conversion & \multicolumn{4}{c}{ Yield (\%) } \\
\cline { 3 - 5 } & $(\%)$ & $\mathbf{1 6}$ & $\mathbf{1 7}$ & $\mathbf{1 8}$ \\
\hline 1 & 68 & 17 & 81 & 1 \\
3 & $>99$ & 26 & 72 & 2 \\
\hline
\end{tabular}




\subsubsection{NMR Spectroscopic Data for 16-18}

Selected NMR spectroscopic data for 16: ${ }^{1} \mathrm{H}$ NMR $\left(500 \mathrm{MHz}, \mathrm{C}_{6} \mathrm{D}_{6}\right): \delta / p p m=7.74(\mathrm{~s}, 1 \mathrm{H})$.

NMR spectroscopic data for 17: ${ }^{1} \mathrm{H}$ NMR $\left(500 \mathrm{MHz}, \mathrm{C}_{6} \mathrm{D}_{6}\right): \delta / p p m=0.65$ (q, J = $\left.8.0 \mathrm{~Hz}, 12 \mathrm{H}\right)$, $1.03(\mathrm{t}, J=8.0 \mathrm{~Hz}, 18 \mathrm{H}), 5.06(\mathrm{~s}, 2 \mathrm{H}) .{ }^{13} \mathrm{C}\left\{{ }^{1} \mathrm{H}\right\} \mathrm{NMR}\left(126 \mathrm{MHz}, \mathrm{C}_{6} \mathrm{D}_{6}\right): \delta / p p m=5.2(\mathrm{~s}, 6 \mathrm{C})$, 7.0 (s, 6C), 84.5 (s). ${ }^{1} \mathrm{H},{ }^{29} \mathrm{Si}$ HMQC NMR $\left(500 \mathrm{MHz} / 99 \mathrm{MHz}, \mathrm{C}_{6} \mathrm{D}_{6}\right): \delta / p p m=0.65 / 18.5$, 1.03/18.5, 5.06/18.5.

Selected NMR spectroscopic data for 18: ${ }^{1} \mathrm{H}$ NMR $\left(500 \mathrm{MHz}, \mathrm{C}_{6} \mathrm{D}_{6}\right): \delta / p p m=3.31(\mathrm{~s}, 3 \mathrm{H})$. The spectroscopic data are in accordance with those reported. ${ }^{[\mathrm{S} 11]}$

\subsection{1-Dimethylphenylsilyl-1,4-dihydropyridine (20)}

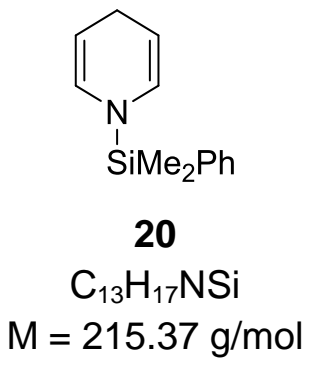

\subsubsection{Synthesis of 20 Using Dimeric Ruthenium NHC Complex 4}

In a glove box, ruthenium dimer 4 (3.6 mg, $1.3 \mu \mathrm{mol}, 0.50 \mathrm{~mol} \%$ ) was weighed into a GLC vial. Pyridine $(19,20 \mathrm{mg}, 0.25 \mathrm{mmol}, 1.0$ equiv) and dimethylphenylsilane (34 mg, $0.25 \mathrm{mmol}, 1.0$ equiv) were added, and the resulting mixture was stirred at room temperature for $17 \mathrm{~h}$. The reaction mixture was diluted and filtered through a small pad of Celite ${ }^{\circledR}$ layered with a small amount of silica gel using a solution of cyclohexane, tert-butylmethyl ether and triethylamine (80:5:1, $0.5 \mathrm{~mL}+5 \mathrm{~mL})$. Evaporation of the solvents under reduced pressure and drying in high vacuum afforded dihydropyridine 20 (47 mg, 87\%) as a yellowish, viscous oil.

\subsubsection{Synthesis of 20 Using Ruthenium NHC Chloride Complex 7 and $\mathrm{NaBAr}_{4}$}

In a glove box, ruthenium chloride $7(1.5 \mathrm{mg}, 2.5 \mu \mathrm{mol}, 1.0 \mathrm{~mol} \%)$ and $\mathrm{NaBAr}_{4}(2.2 \mathrm{mg}$, $2.5 \mu \mathrm{mol}, 1.0 \mathrm{~mol} \%)$ were weighed into a GLC vial. Pyridine (19, $20 \mathrm{mg}, 0.25 \mathrm{mmol}, 1.0$ equiv) and dimethylphenylsilane (34 mg, $0.25 \mathrm{mmol}, 1.0$ equiv) were added, and the resulting mixture was stirred at room temperature for $17 \mathrm{~h}$. The reaction mixture was diluted and filtered through a small pad of Celite ${ }^{\circledR}$ layered with a small amount of silica gel using a solution of cyclohexane, tert-butylmethyl ether and triethylamine $(80: 5: 1,0.5 \mathrm{~mL}+5 \mathrm{~mL})$. Evaporation of the solvents under reduced pressure and drying in high vacuum afforded dihydropyridine 20 (53 mg, 98\%) as a yellowish, viscous oil.

\subsubsection{NMR Spectroscopic Data for 20}

${ }^{1} \mathrm{H}$ NMR $\left(500 \mathrm{MHz}, \mathrm{C}_{6} \mathrm{D}_{6}\right): \delta / \mathrm{ppm}=0.18(\mathrm{~s}, 6 \mathrm{H}), 3.04-3.79(\mathrm{~m}, 2 \mathrm{H}), 4.50(\mathrm{dt}, J=8.5,3.2 \mathrm{~Hz}$, $2 \mathrm{H}), 5.90(\mathrm{dt}, J=8.5,1.4 \mathrm{~Hz}, 2 \mathrm{H}), 7.13-7.16(\mathrm{~m}, 3 \mathrm{H}), 7.44-7.46(\mathrm{~m}, 2 \mathrm{H}) .{ }^{13} \mathrm{C}\left\{{ }^{1} \mathrm{H}\right\}$ NMR $\left(126 \mathrm{MHz}, \mathrm{C}_{6} \mathrm{D}_{6}\right): \delta / \mathrm{ppm}=-2.5(\mathrm{~s}, 2 \mathrm{C}), 23.2(\mathrm{~s}), 100.6(\mathrm{~s}, 2 \mathrm{C}), 128.3(\mathrm{~s}, 2 \mathrm{C}), 129.1(\mathrm{~s}, 2 \mathrm{C})$, 130.0 (s), 134.2 (s, 2C), 136.9 (s). ${ }^{29} \mathrm{Si}\left\{{ }^{1} \mathrm{H}\right\}$ DEPT NMR (99 MHz, $\left.\mathrm{C}_{6} \mathrm{D}_{6}\right): \delta / p p m=2.2$. The spectroscopic data are in accordance with those reported. ${ }^{[\mathrm{S12]}}$ 


\section{$4 \quad$ Mechanistic Experiments}

\subsection{Partial Formation of Monomeric Ruthenium NHC Complex 8 at Elevated} Temperatures

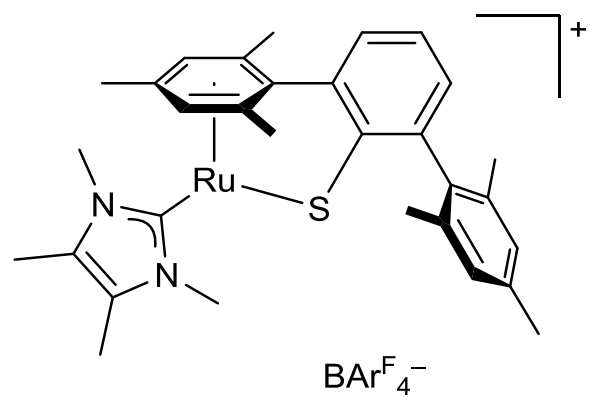

8

$$
\begin{gathered}
\mathrm{C}_{63} \mathrm{H}_{49} \mathrm{BF}_{24} \mathrm{~N}_{2} \mathrm{RuS} \\
\mathrm{M}=1434.00 \mathrm{~g} / \mathrm{mol}
\end{gathered}
$$

A solution of ruthenium dimer $4(5.6 \mathrm{mg}, 2.0 \mu \mathrm{mol})$ in $0-\mathrm{Cl}_{2} \mathrm{C}_{6} \mathrm{D}_{4}(0.5 \mathrm{~mL})$ was transferred to an NMR tube. The sample was directly submitted to NMR spectroscopic analysis. ${ }^{1} \mathrm{H}$ NMR spectra were subsequently recorded at room temperature, $60^{\circ} \mathrm{C}$ and $80^{\circ} \mathrm{C}$. A control spectrum was recorded after recooling the sample to room temperature. The experiments indicated the partial and reversible formation of monomer 8 at temperatures of $60{ }^{\circ} \mathrm{C}$ and above.

Selected NMR spectroscopic data for 8: ${ }^{1} \mathbf{H}$ NMR $\left(500 \mathrm{MHz}, \quad o-\mathrm{Cl}_{2} \mathrm{C}_{6} \mathrm{D}_{4}\right): \delta / p p m=1.80(\mathrm{~s}$, $6 \mathrm{H}), 1.81(\mathrm{~s}, 6 \mathrm{H}), 1.94(\mathrm{~s}, 3 \mathrm{H}), 1.98(\mathrm{~s}, 6 \mathrm{H}), 3.09(\mathrm{~s}, 6 \mathrm{H}), 4.58(\mathrm{~s}, 2 \mathrm{H})$.

1: r.t.

2: $60^{\circ} \mathrm{C}$

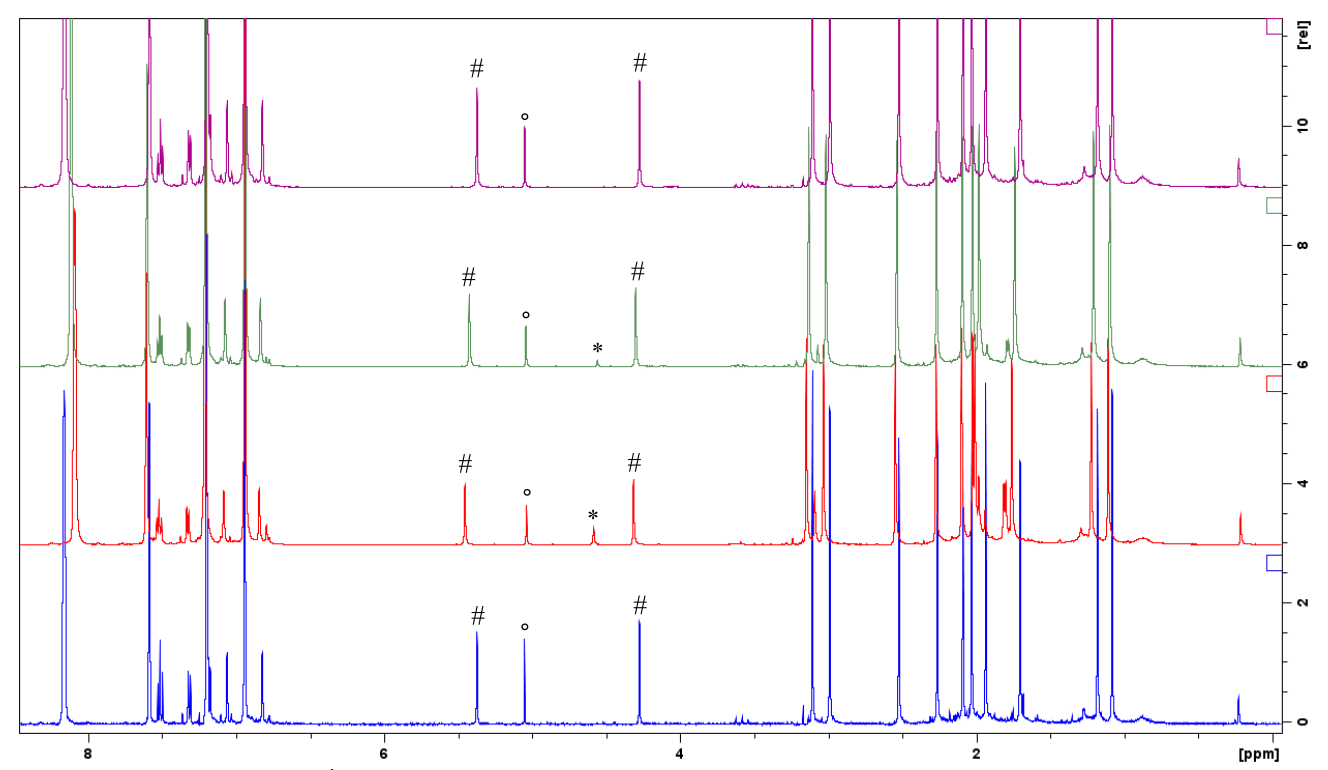

4: recooled to r.t.

Figure S3. Variable temperature ${ }^{1} \mathrm{H}$ NMR analysis $\left(500 \mathrm{MHz}, \mathrm{o}_{-}-\mathrm{Cl}_{2} \mathrm{C}_{6} \mathrm{D}_{4}\right)$ of the equilibrium between dimeric complex 4 and monomeric complex 8. \# = Dimeric NHC ruthenium complex $4 ; *=$ monomeric ruthenium $\mathrm{NHC}$ complex 8; ${ }^{\circ}=\mathrm{CH}_{2} \mathrm{Cl}_{2}$. 


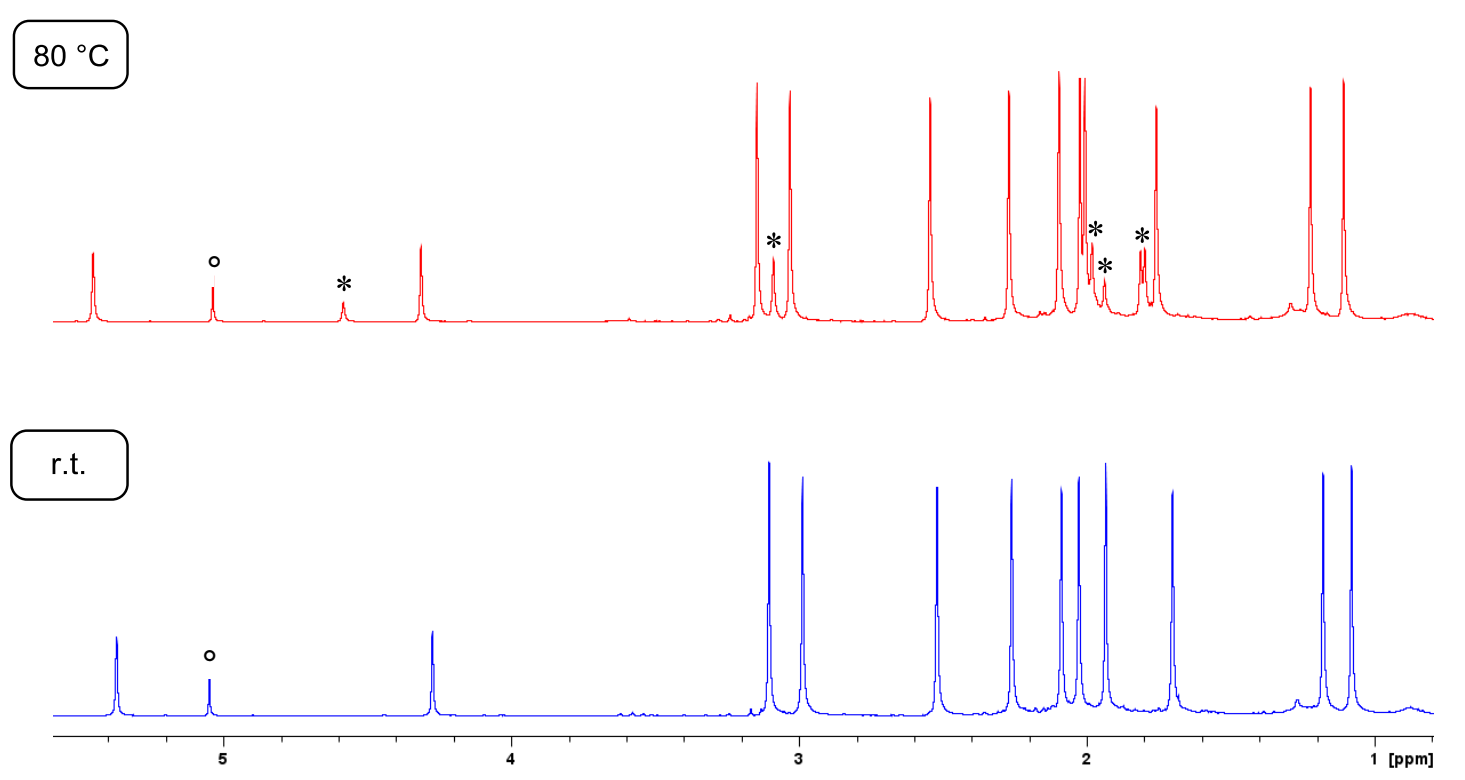

Figure S4. Segments of the variable temperature ${ }^{1} \mathrm{H}$ NMR analysis. Red: Dimeric ruthenium $\mathrm{NHC}$ complex 4 and partially formed monomeric ruthenium NHC complex $8(*)$; blue: dimeric ruthenium $\mathrm{NHC}$ complex 4; $\circ=\mathrm{CH}_{2} \mathrm{Cl}_{2}$.

\subsection{Partial Formation of Pyridine-Coordinated Monomeric Complex 8-py}

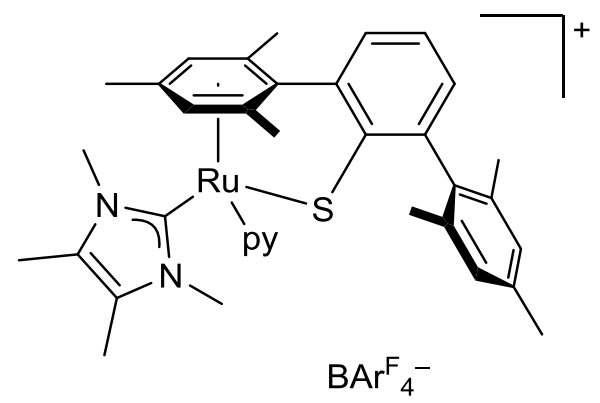

$$
\begin{gathered}
\text { 8·py } \\
\mathrm{C}_{68} \mathrm{H}_{54} \mathrm{BF}_{24} \mathrm{~N}_{3} \mathrm{RuS} \\
\mathrm{M}=1513.10 \mathrm{~g} / \mathrm{mol}
\end{gathered}
$$

In an NMR tube, a solution of pyridine (19) in $\mathrm{CD}_{2} \mathrm{Cl}_{2}(0.20 \mathrm{M}, 0.10 \mathrm{~mL}, 20 \mu \mathrm{mol}, 10$ equiv) was added to a solution of ruthenium dimer $4\left(5.6 \mathrm{mg}, 2.0 \mu \mathrm{mol}, 1.0\right.$ equiv) in $\mathrm{CD}_{2} \mathrm{Cl}_{2}(0.4$ $\mathrm{mL})$. The mixture was maintained at room temperature for $4 \mathrm{~d}$. ${ }^{1} \mathrm{H}$ NMR spectroscopic experiments indicated the partial formation of pyridine-coordinated ruthenium complex 8.py along with unreacted ruthenium dimer 4 ( 1:3 ratio of 8.py to 4) and pyridine (19). The coordination of pyridine is reversible according to ${ }^{1} \mathrm{H},{ }^{1} \mathrm{H}$ EXSY spectroscopy.

Selected NMR spectroscopic data for 8.py: ${ }^{1} \mathbf{H}$ NMR $\left(500 \mathrm{MHz}, \mathrm{CD}_{2} \mathrm{Cl}_{2}\right): \delta / p p m=1.74(\mathrm{~s}$, $3 \mathrm{H}), 1.91(\mathrm{~s}, 3 \mathrm{H}), 2.02(\mathrm{~s}, 3 \mathrm{H}), 2.09(\mathrm{~s}, 3 \mathrm{H}), 2.17(\mathrm{~s}, 3 \mathrm{H}), 2.28(\mathrm{~s}, 3 \mathrm{H}), 5.00(\mathrm{~s}, 1 \mathrm{H}), 5.71(\mathrm{~s}$, $1 \mathrm{H}), 6.81(\mathrm{~s}, 1 \mathrm{H}), 7.13-7.17(\mathrm{~m}, 2 \mathrm{H}), 7.95\left(\mathrm{~s}_{\mathrm{br}}, 2 \mathrm{H}\right)$.

The signals for the $\mathrm{N}-\mathrm{CH}_{3}$ groups are broadened into the baseline. 

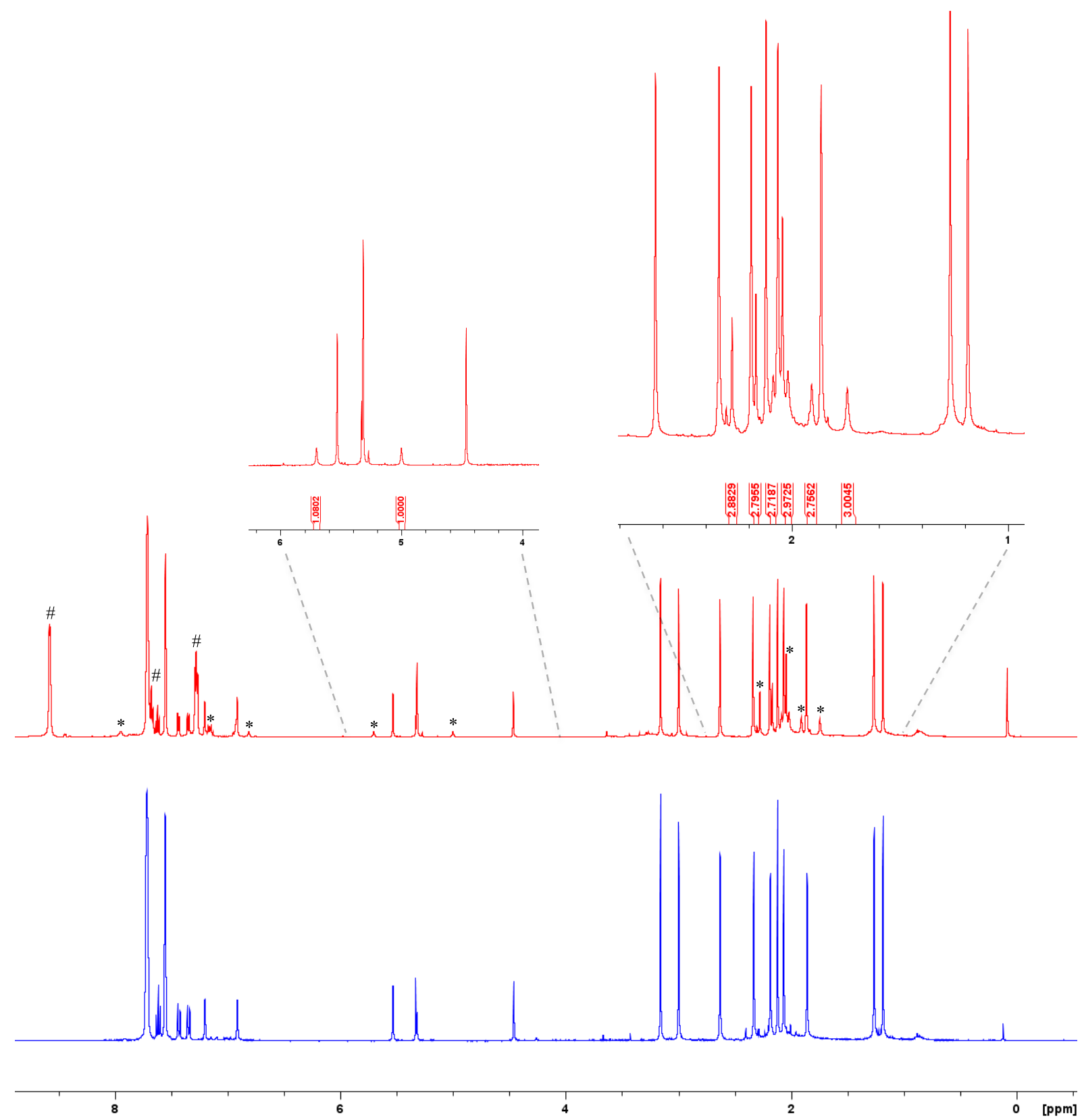

Figure S5. Comparison of the ${ }^{1} \mathrm{H}$ NMR spectra $\left(500 \mathrm{MHz}, \mathrm{CD}_{2} \mathrm{Cl}_{2}\right)$ of partially formed complex 8-pyridine and dimeric complex 4. Red: mixture of dimeric ruthenium NHC complex 4 and pyridine $(19, \#)$, resulting in the partial formation of 8 pyridine $(*)$; blue: dimeric ruthenium NHC complex 4. 


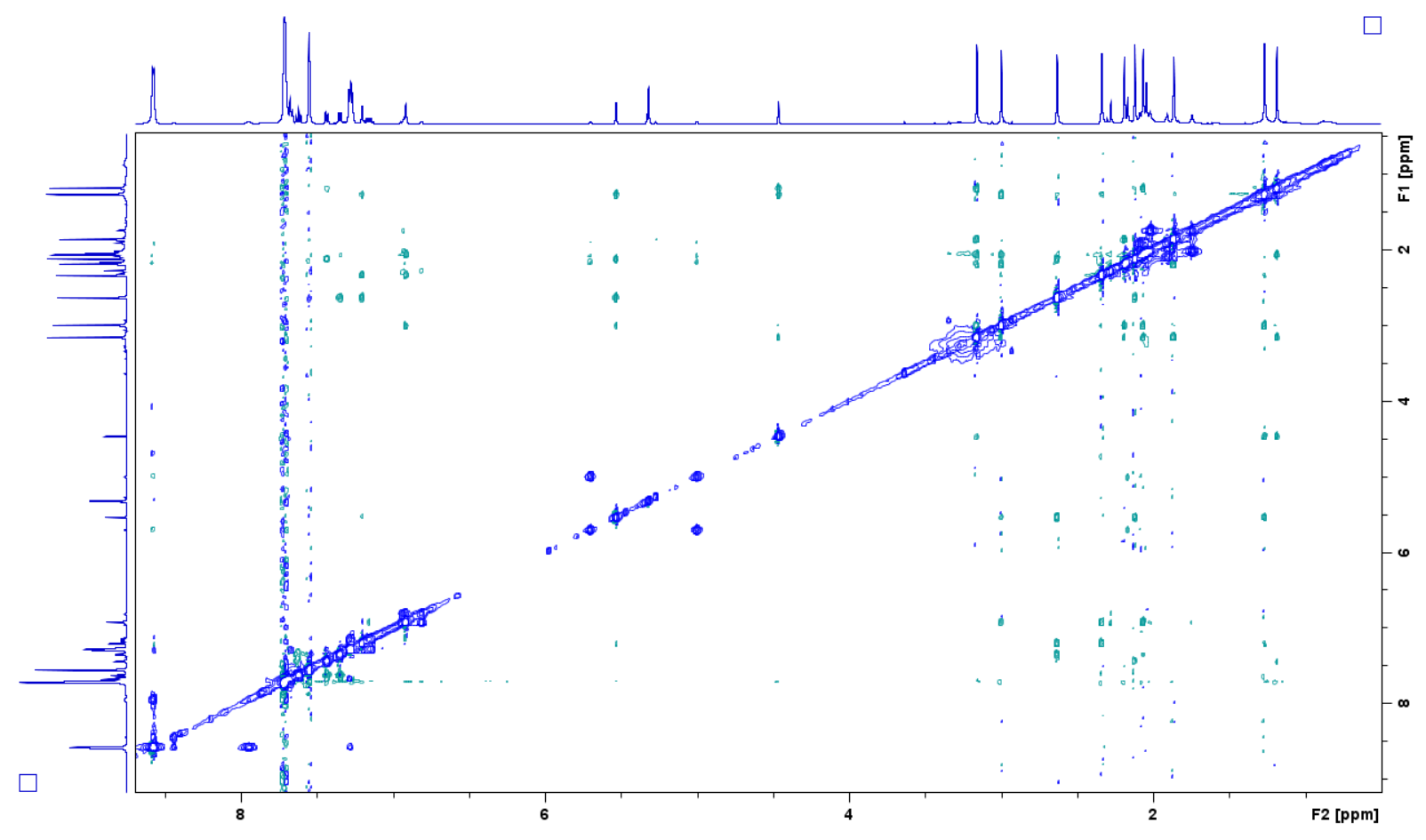

\section{Partial View:}

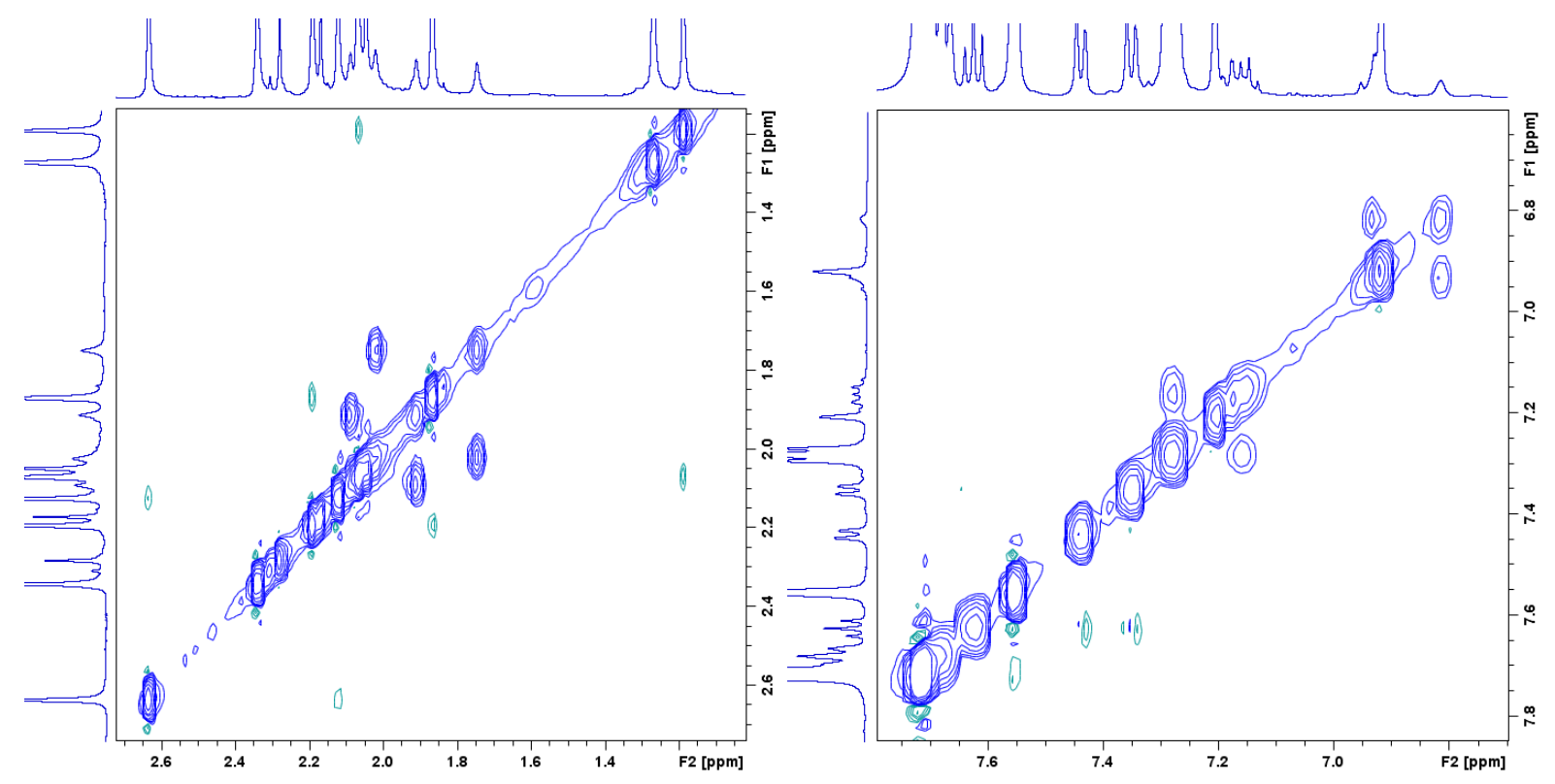

Figure S6. ${ }^{1} \mathrm{H},{ }^{1} \mathrm{H}$ EXSY NMR $\left(500 / 500 \mathrm{MHz}, \mathrm{CD}_{2} \mathrm{Cl}_{2}, 300 \mathrm{~K}, \mathrm{~T}_{\mathrm{m}}=600 \mathrm{~ms}\right)$ of a mixture of dimeric ruthenium NHC complex 4 and pyridine (19), resulting in the partial formation of 8. pyridine. Green = normal phase, blue = negative phase. 


\section{$5 \quad$ NMR Spectra}

Ruthenium NHC Chloride Complex 7

${ }^{1} \mathrm{H}$ NMR $\left(400 \mathrm{MHz}, \mathrm{CD}_{2} \mathrm{Cl}_{2}\right)$ :
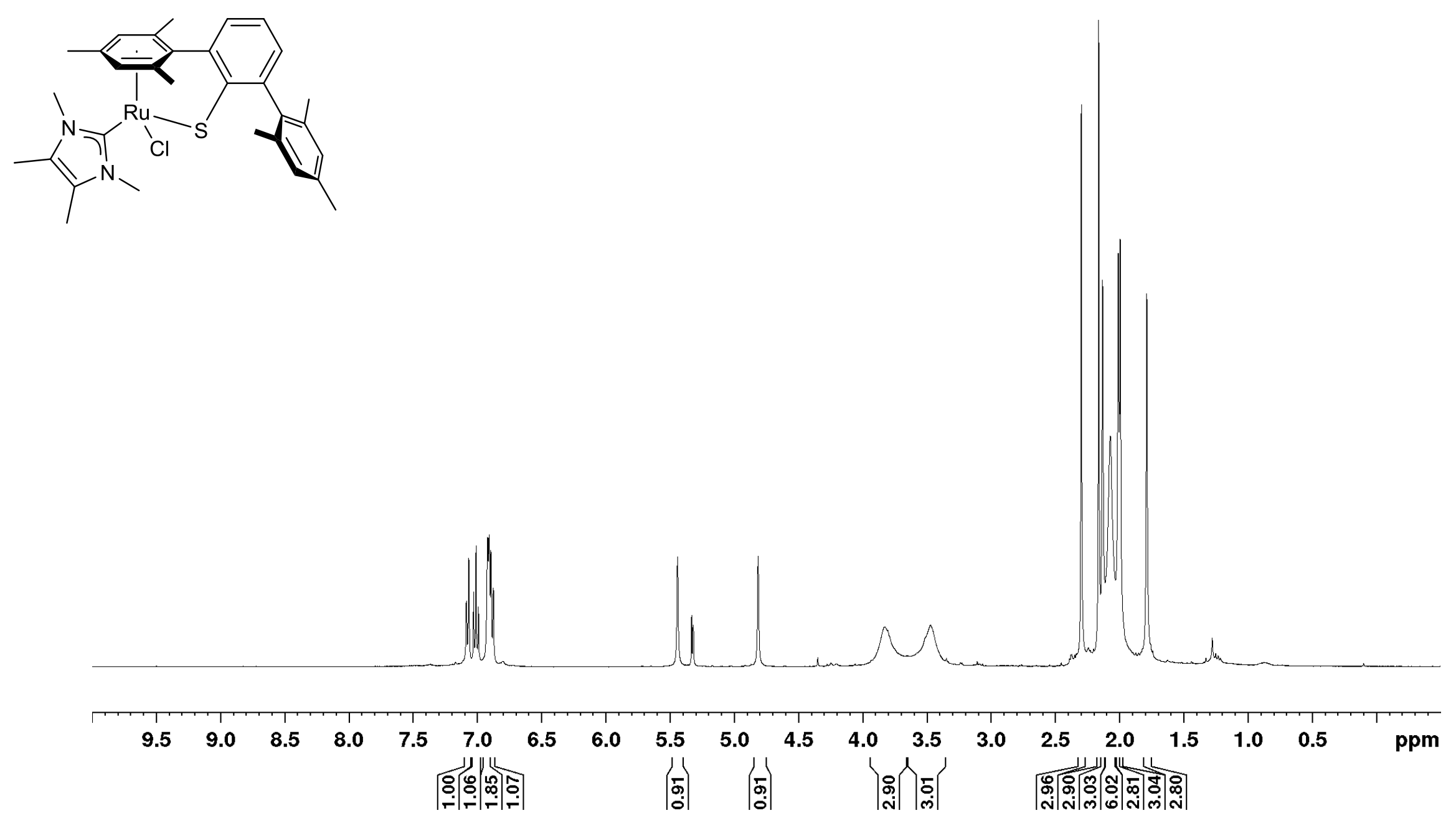
${ }^{13} \mathrm{C}\left\{{ }^{1} \mathrm{H}\right\}$ NMR $\left(126 \mathrm{MHz}, \mathrm{CD}_{2} \mathrm{Cl}_{2}\right):$
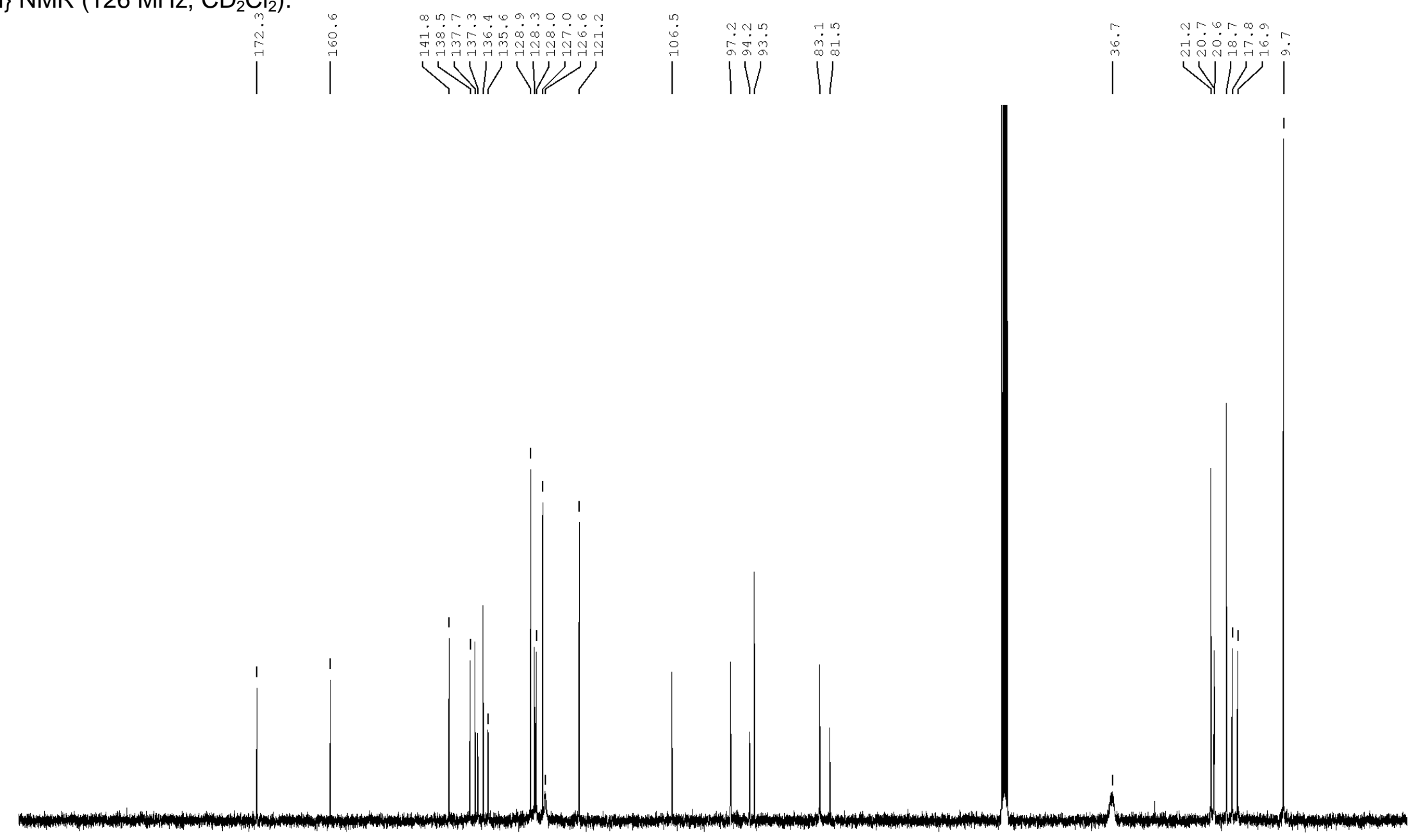


\section{Dimeric Ruthenium NHC Complex 4}

${ }^{1} \mathrm{H}$ NMR $\left(400 \mathrm{MHz}, \mathrm{CD}_{2} \mathrm{Cl}_{2}\right)$ :

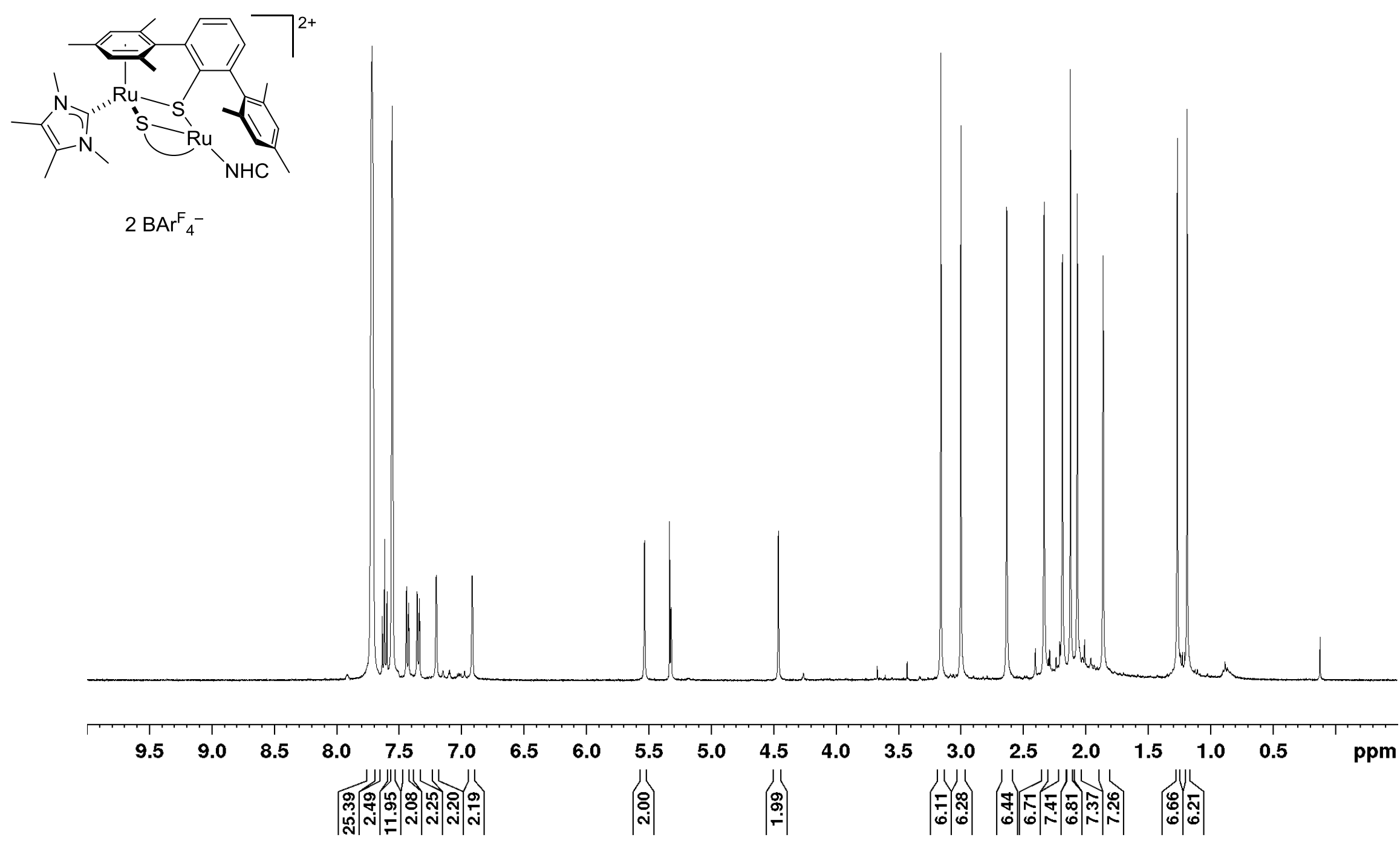


${ }^{11} \mathrm{~B}\left\{{ }^{1} \mathrm{H}\right\} \mathrm{NMR}\left(160 \mathrm{MHz}, \mathrm{CD}_{2} \mathrm{Cl}_{2}\right):$

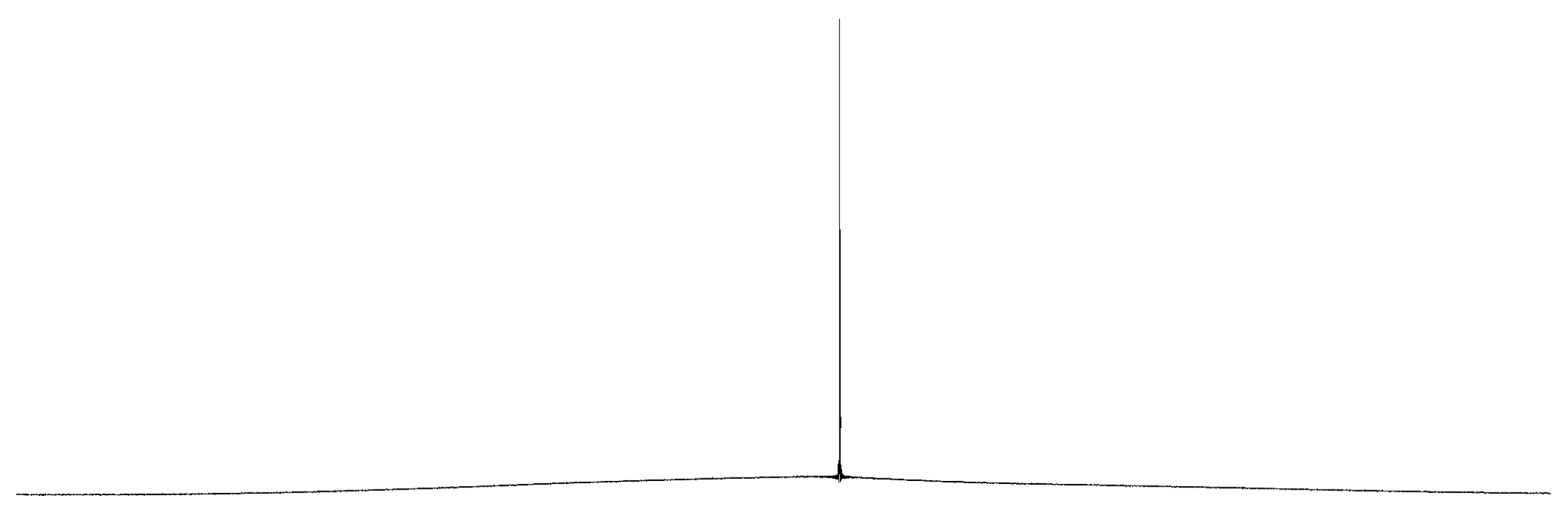


${ }^{13} \mathrm{C}\left\{{ }^{1} \mathrm{H}\right\}$ NMR $\left(101 \mathrm{MHz}, \mathrm{CD}_{2} \mathrm{Cl}_{2}\right):$

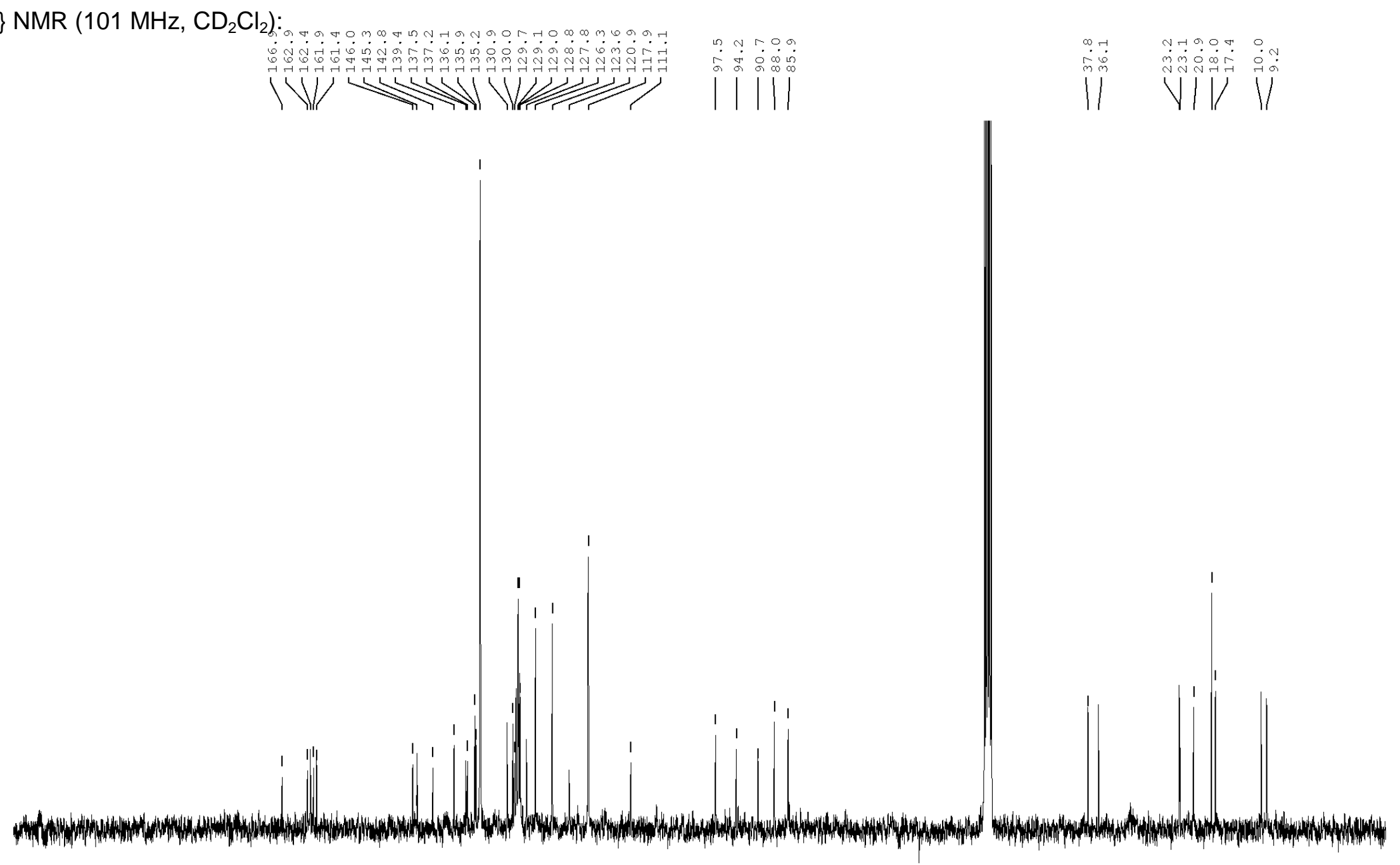

$\begin{array}{llllllllllll}200 & 190 & 180 & 170 & 160 & 150 & 140 & 130 & 120 & 110 & 100 & 90\end{array}$ 
${ }^{19} \mathrm{~F}\left\{{ }^{1} \mathrm{H}\right\} \operatorname{NMR}\left(471 \mathrm{MHz}, \mathrm{CD}_{2} \mathrm{Cl}_{2}\right):$

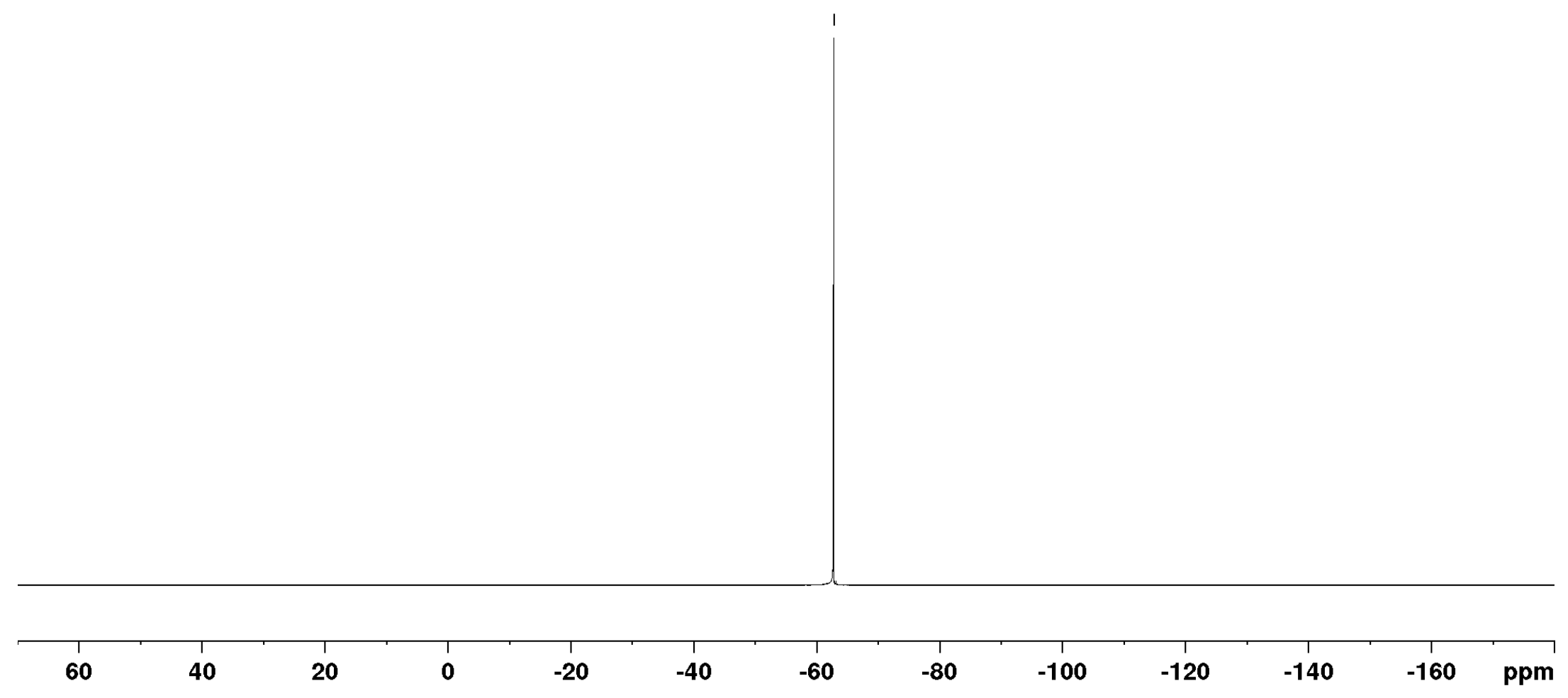


${ }^{1} \mathrm{H},{ }^{1} \mathrm{H}$ NOESY NMR $\left(700 / 700 \mathrm{MHz}, \mathrm{CD}_{2} \mathrm{Cl}_{2}, 300 \mathrm{~K}, \mathrm{~T}_{\mathrm{m}}=600 \mathrm{~ms}\right)$ :

Green $=$ normal phase, blue $=$ negative phase

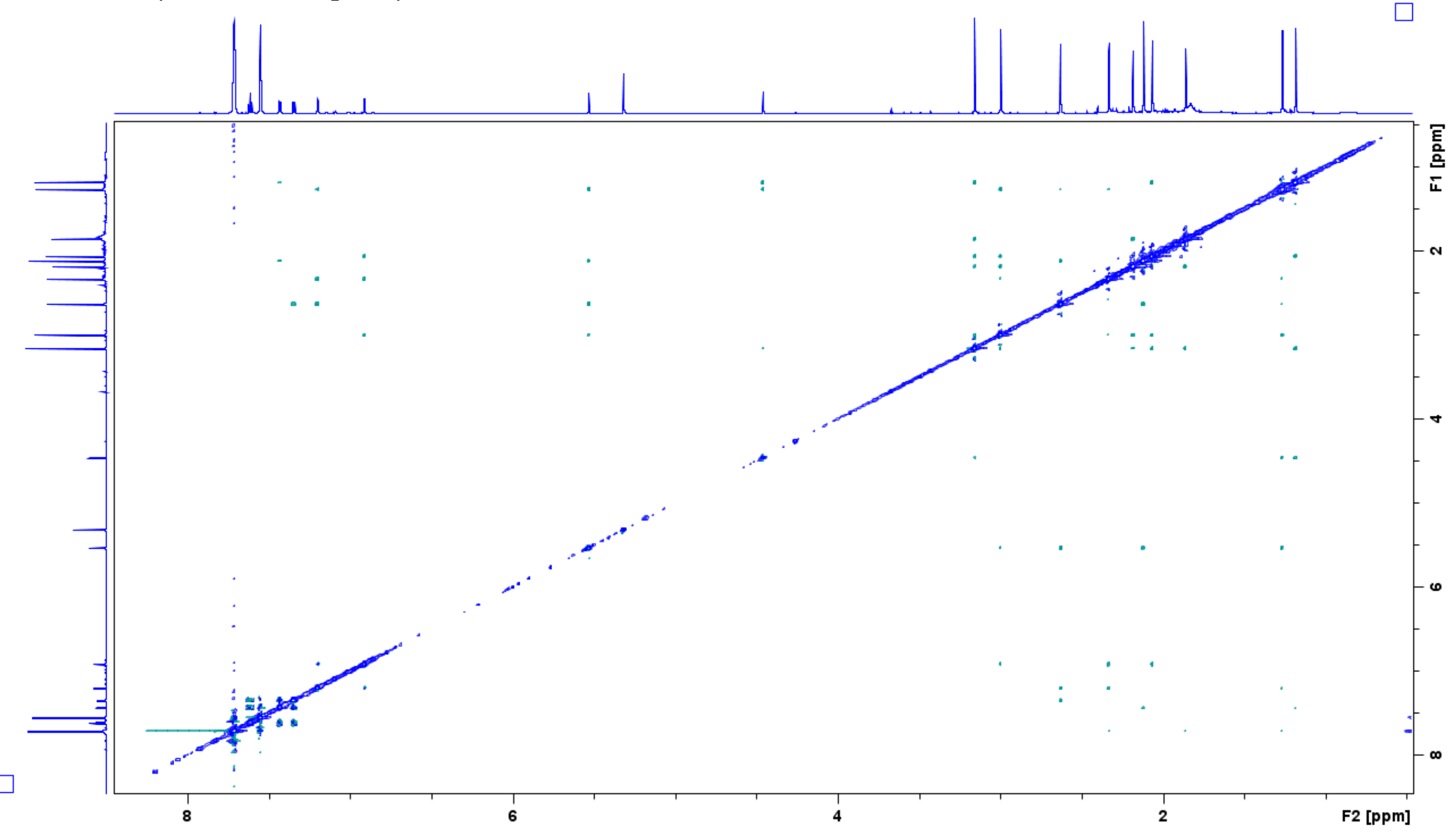




\section{Ethyldimethyl[(1-phenylvinyl)oxy]silane (10) and ethyldimethyl(1-phenylethoxy)silane (11)}

${ }^{1} \mathrm{H}$ NMR $\left(500 \mathrm{MHz}, \mathrm{CDCl}_{3}\right)$ :
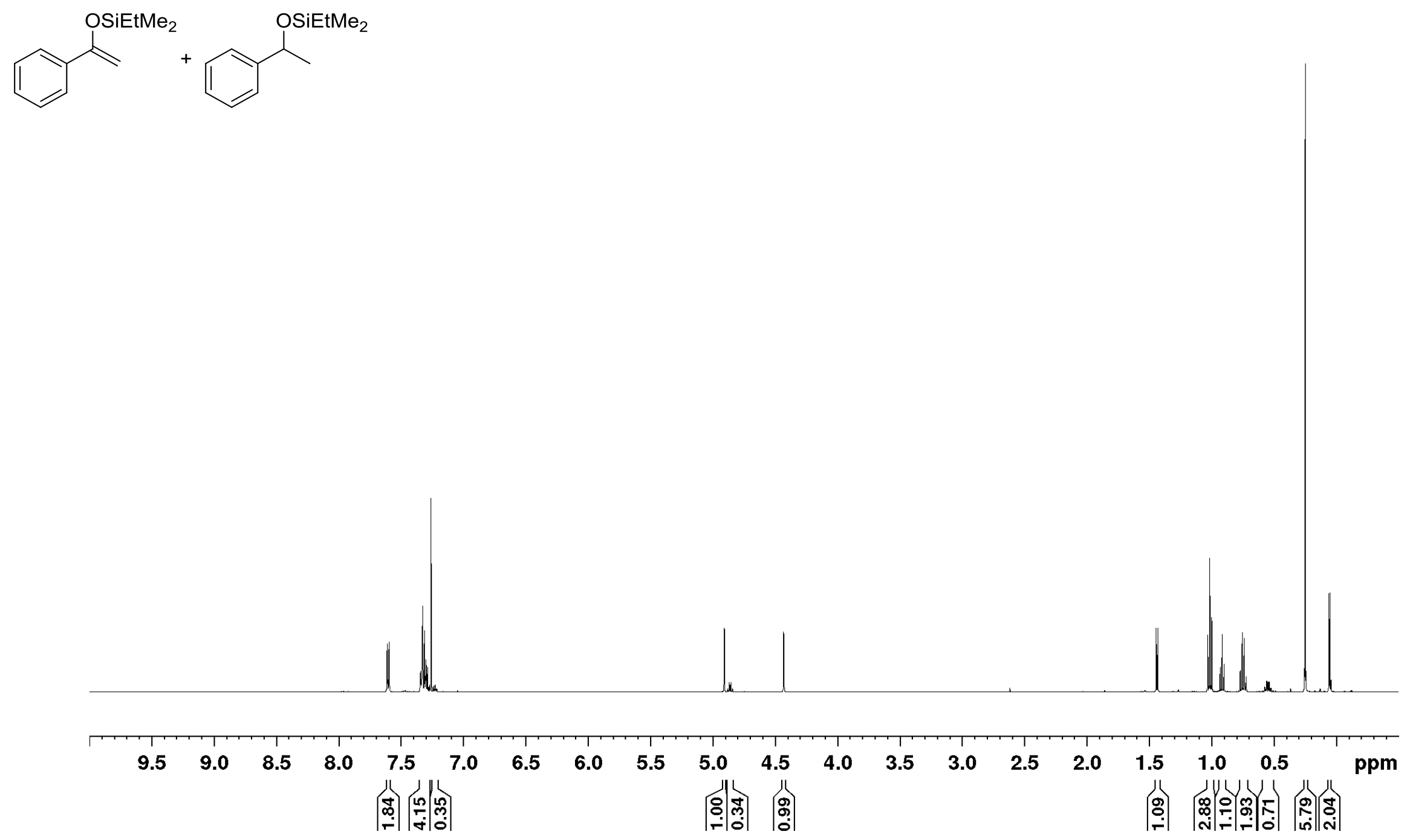
${ }^{13} \mathrm{C}\left\{{ }^{1} \mathrm{H}\right\}$ NMR (126 MHz, $\left.\mathrm{CDCl}_{3}\right):$

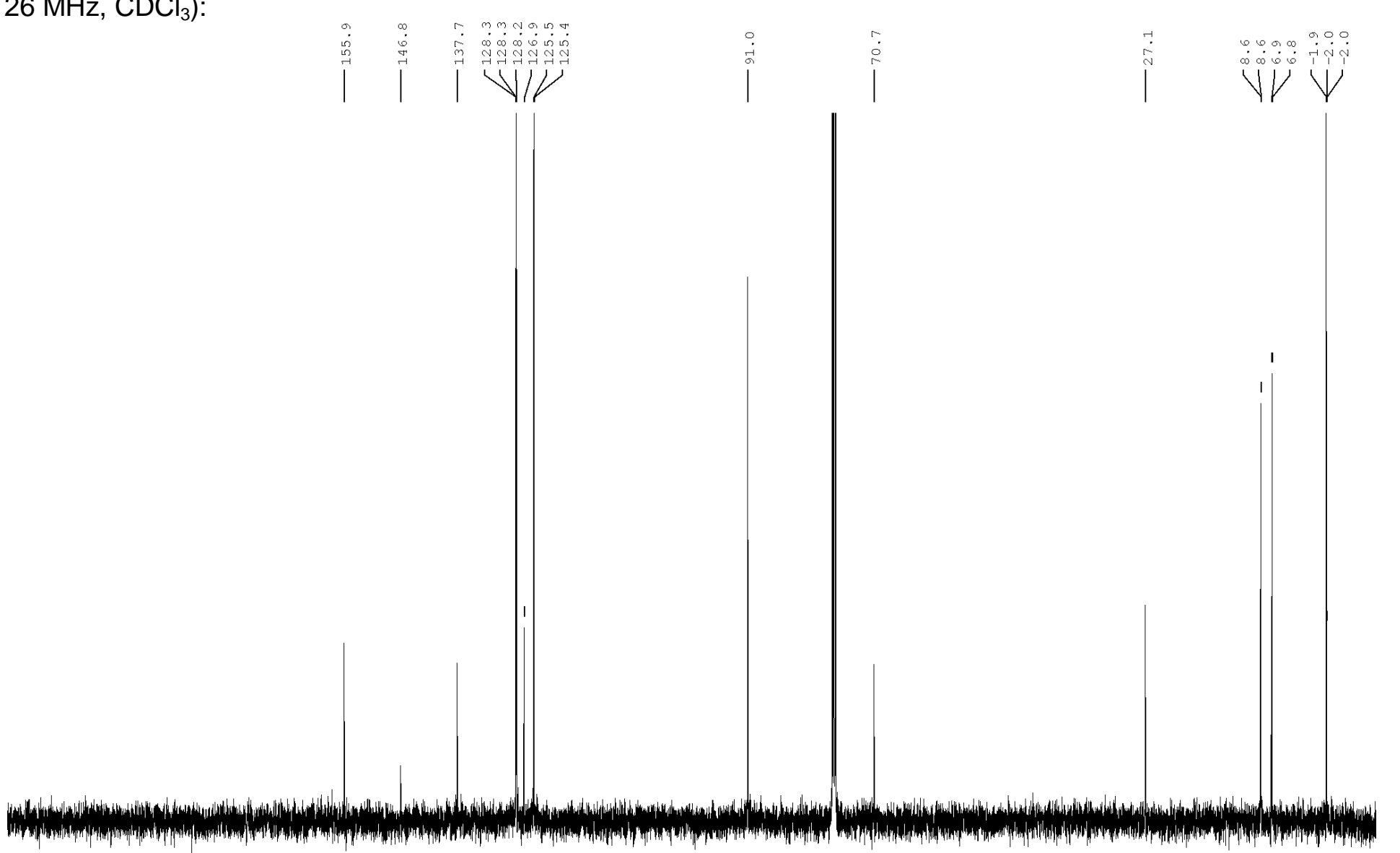

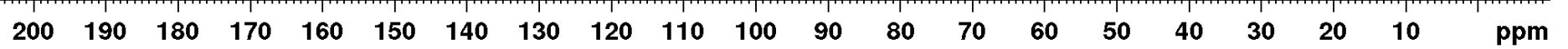


${ }^{29} \mathrm{Si}\left\{{ }^{1} \mathrm{H}\right\}$ DEPT NMR $\left(99 \mathrm{MHz}, \mathrm{CDCl}_{3}\right)$ :

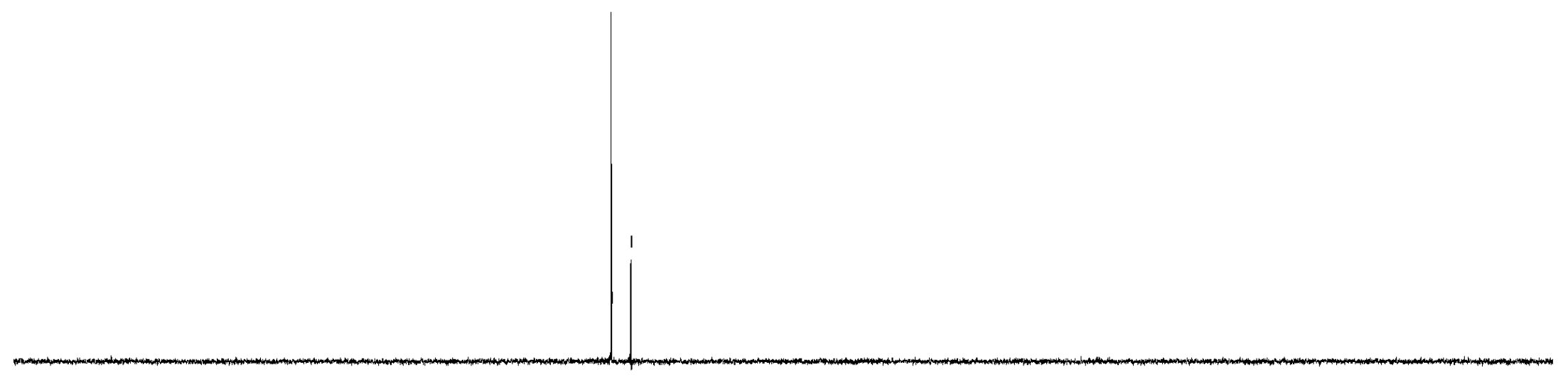

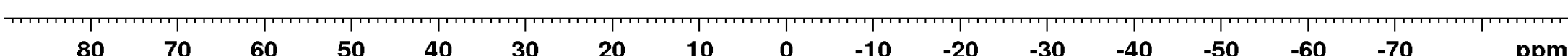


3-Dimethylphenylsilyl-1-methyl-1 $\boldsymbol{H}$-indole (13)

${ }^{1} \mathrm{H}$ NMR $\left(500 \mathrm{MHz}, \mathrm{CDCl}_{3}\right)$ :
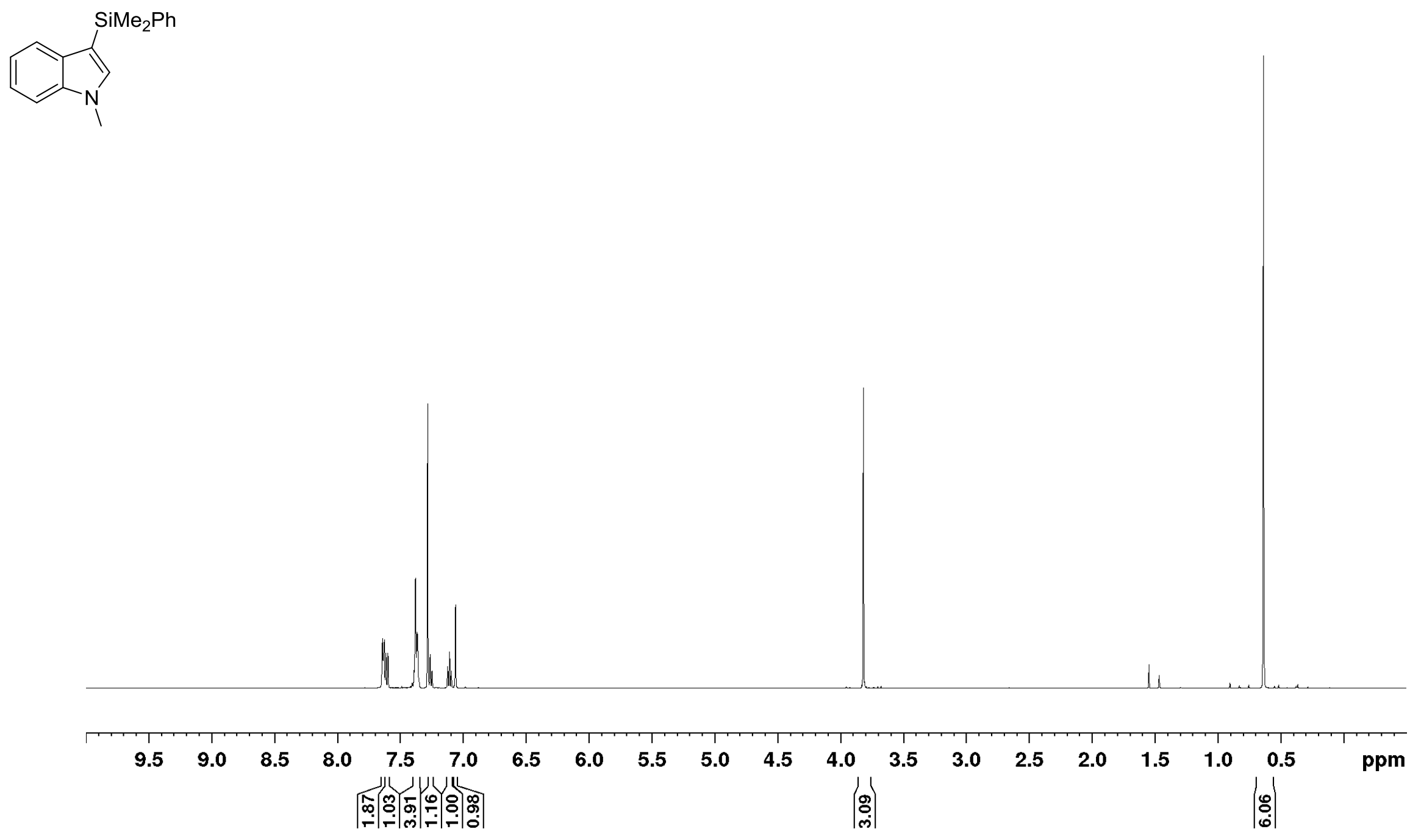
${ }^{13} \mathrm{C}\left\{{ }^{1} \mathrm{H}\right\} \operatorname{NMR}\left(126 \mathrm{MHz}, \mathrm{CDCl}_{3}\right):$
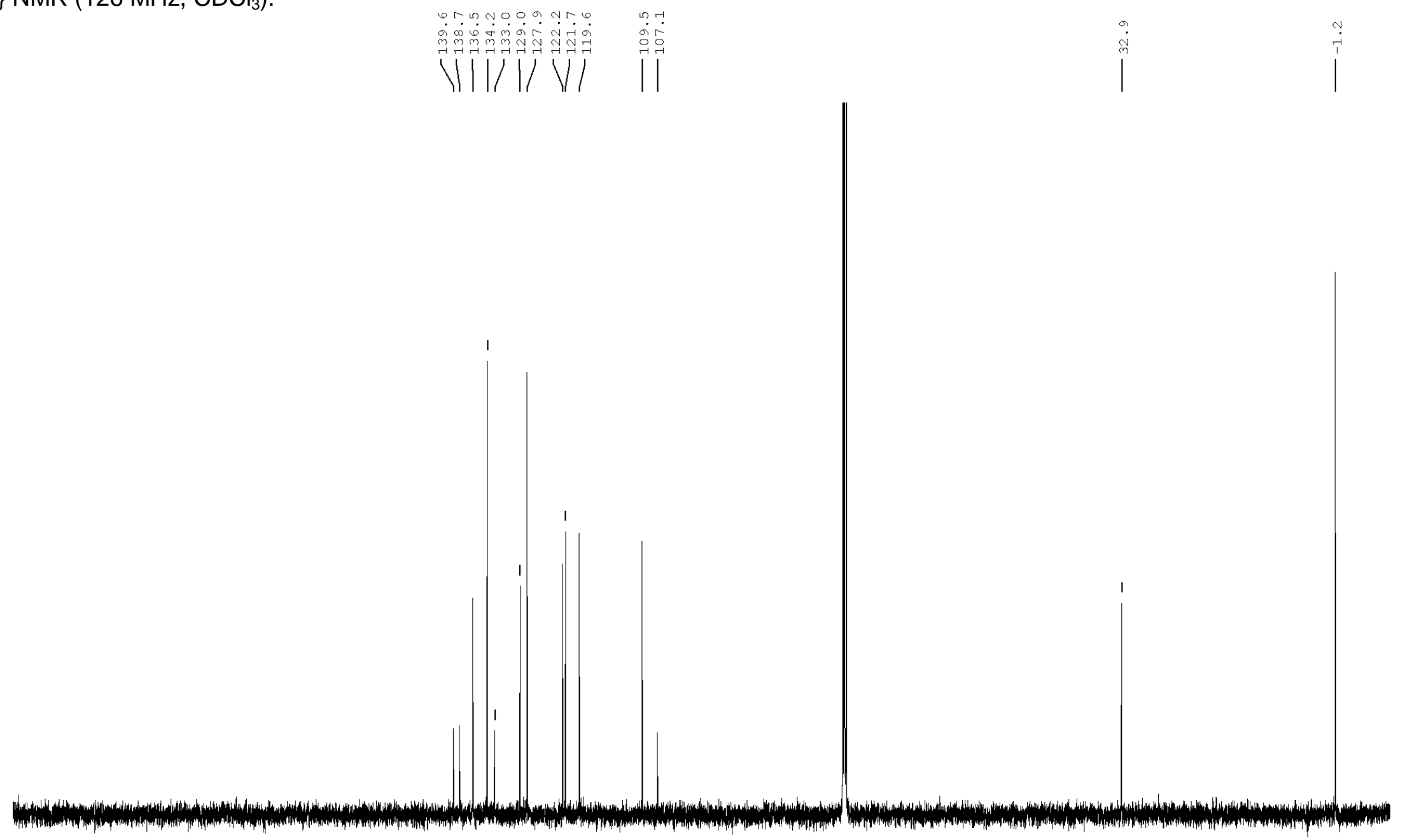
${ }^{29} \mathrm{Si}\left\{{ }^{1} \mathrm{H}\right\}$ DEPT NMR $\left(99 \mathrm{MHz}, \mathrm{CDCl}_{3}\right)$ :

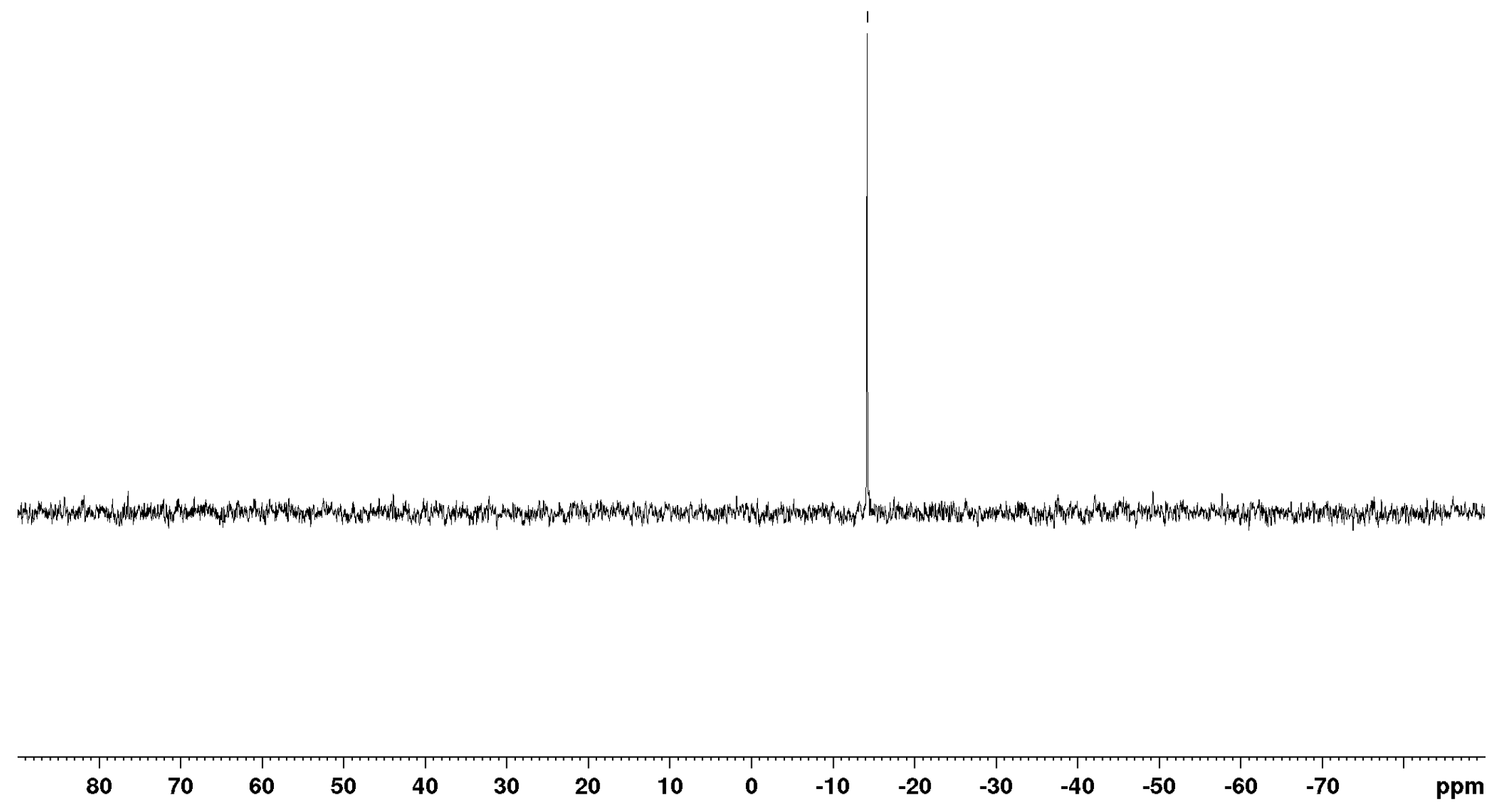


8

8

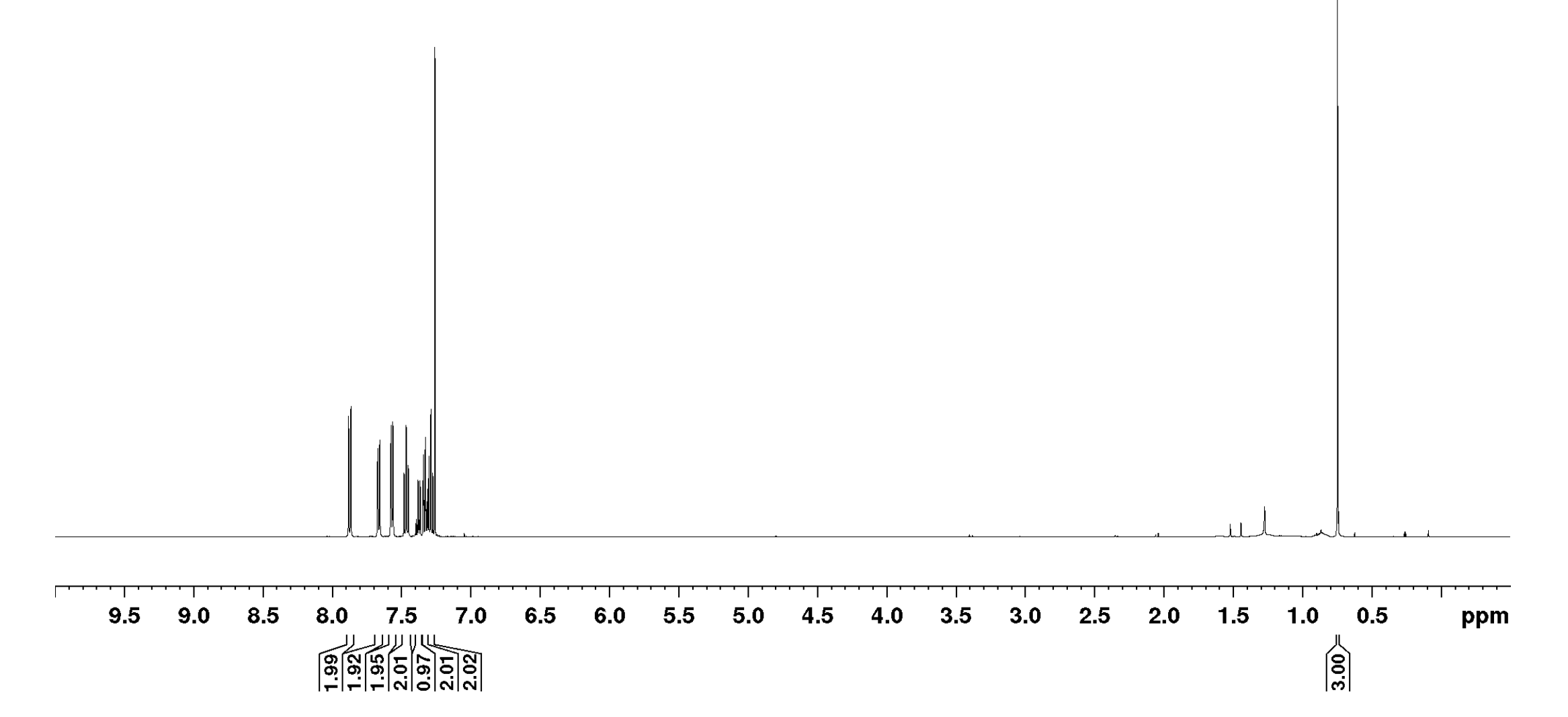


${ }^{13} \mathrm{C}\left\{{ }^{1} \mathrm{H}\right\} \mathrm{NMR}\left(126 \mathrm{MHz}, \mathrm{CDCl}_{3}\right):$
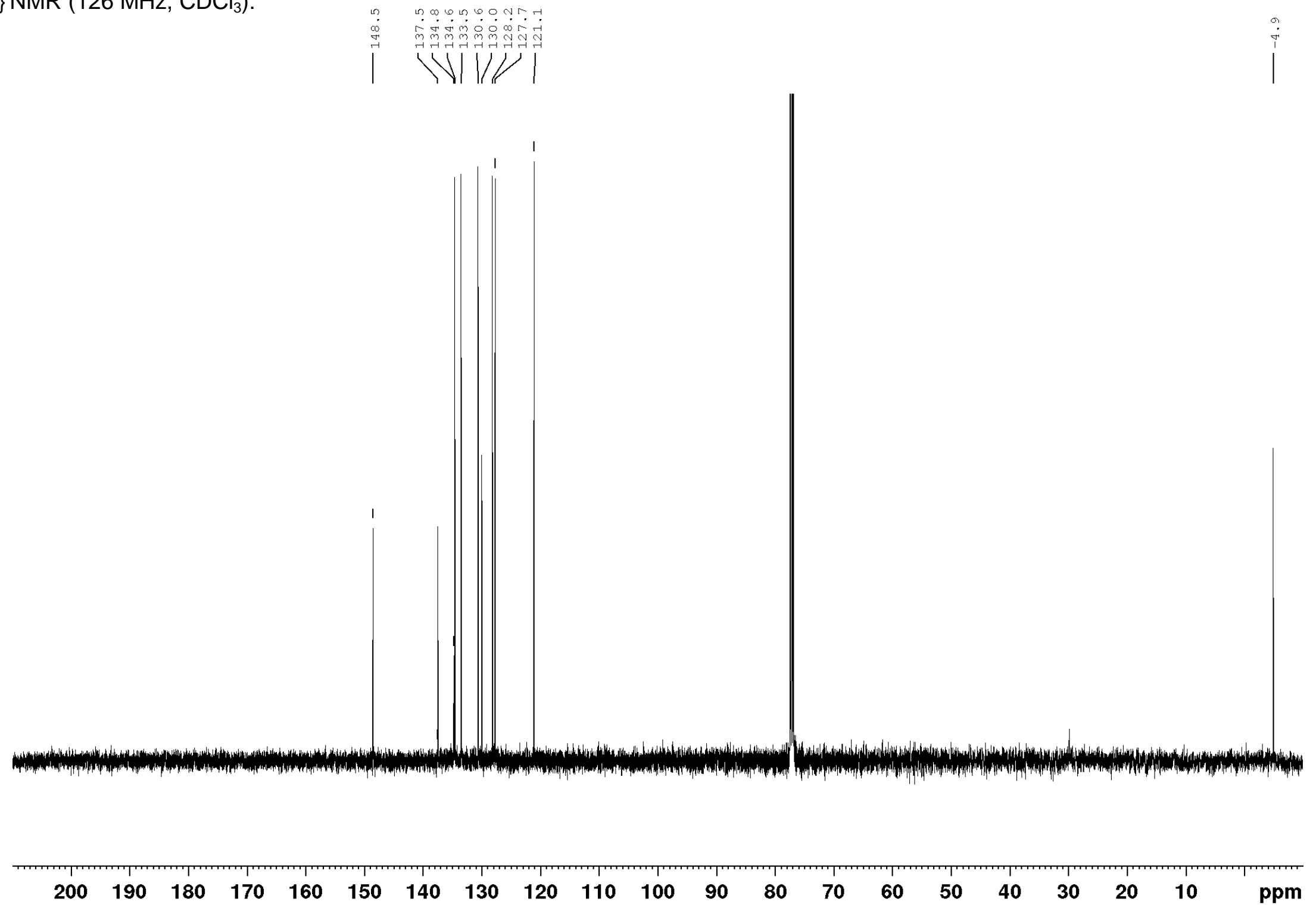
${ }^{29} \mathrm{Si}\left\{{ }^{1} \mathrm{H}\right\}$ DEPT NMR $\left(99 \mathrm{MHz}, \mathrm{CDCl}_{3}\right)$ :

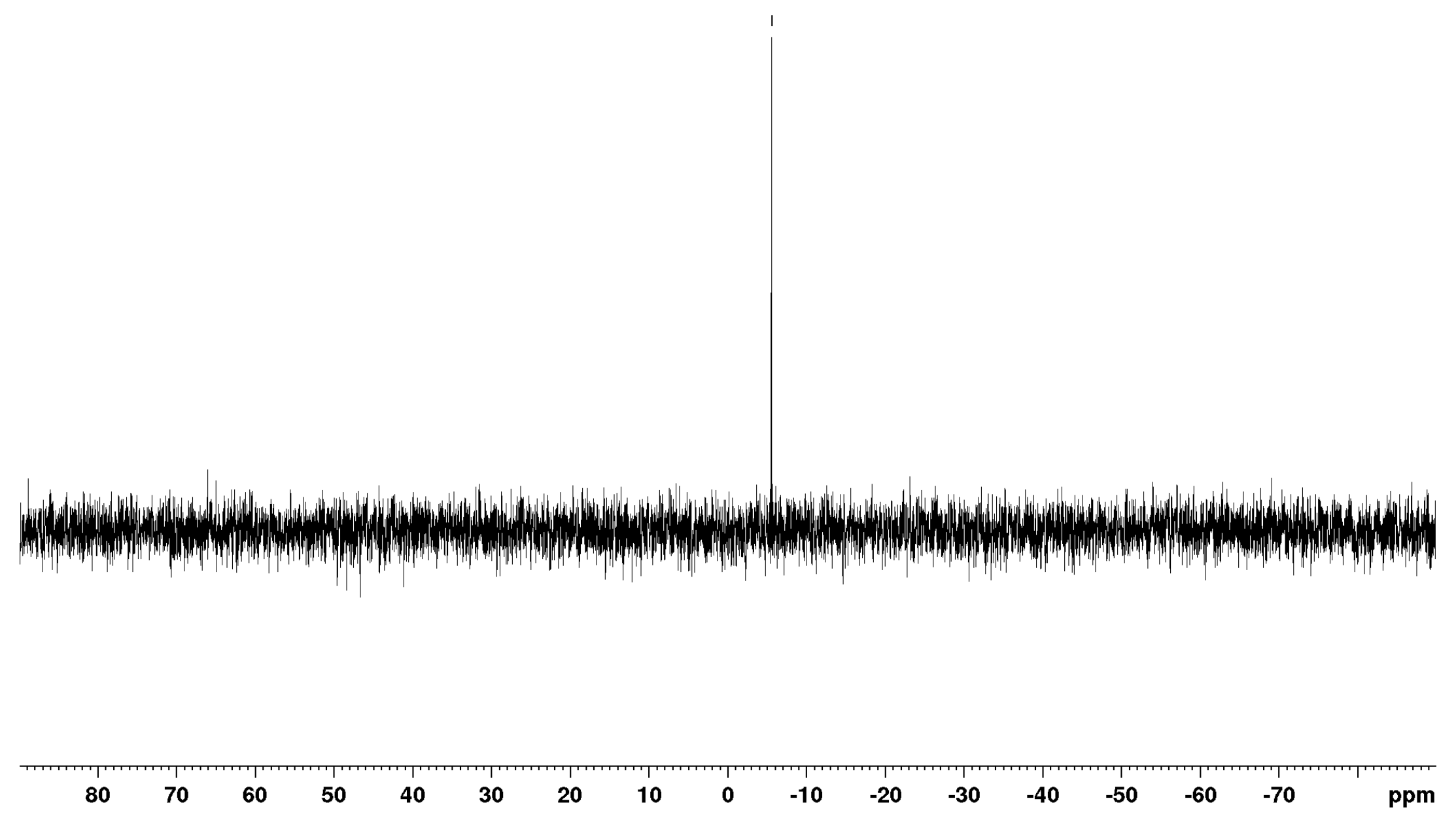




\section{Bis(triethylsilyl)acetal (17)}

${ }^{1} \mathrm{H}$ NMR (500 MHz, $\left.\mathrm{C}_{6} \mathrm{D}_{6}\right)$ :

$\overbrace{\mathrm{H}}^{\mathrm{OSiEt}_{3}}$

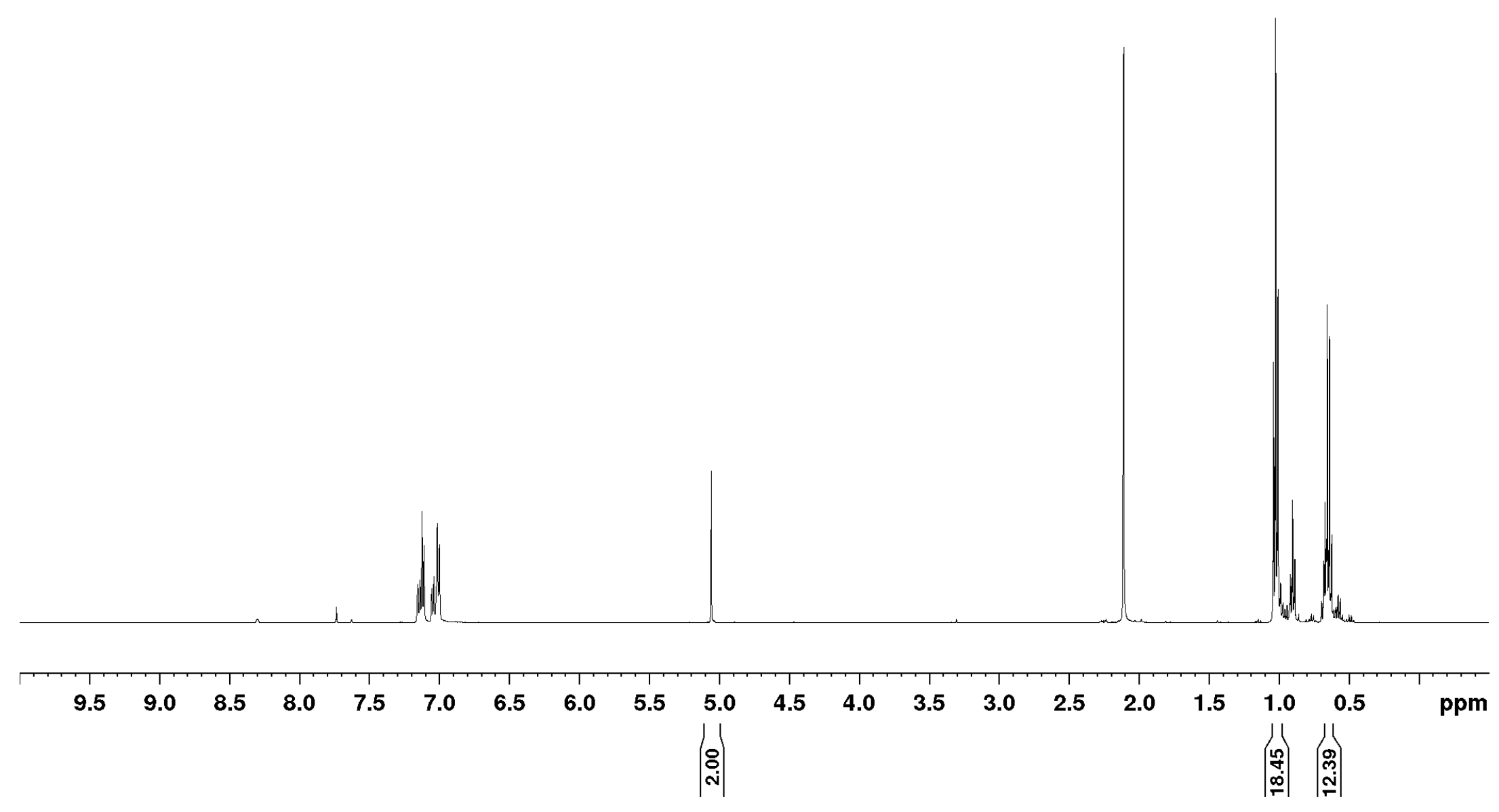


${ }^{13} \mathrm{C}\left\{{ }^{1} \mathrm{H}\right\}$ NMR $\left(126 \mathrm{MHz}, \mathrm{C}_{6} \mathrm{D}_{6}\right):$

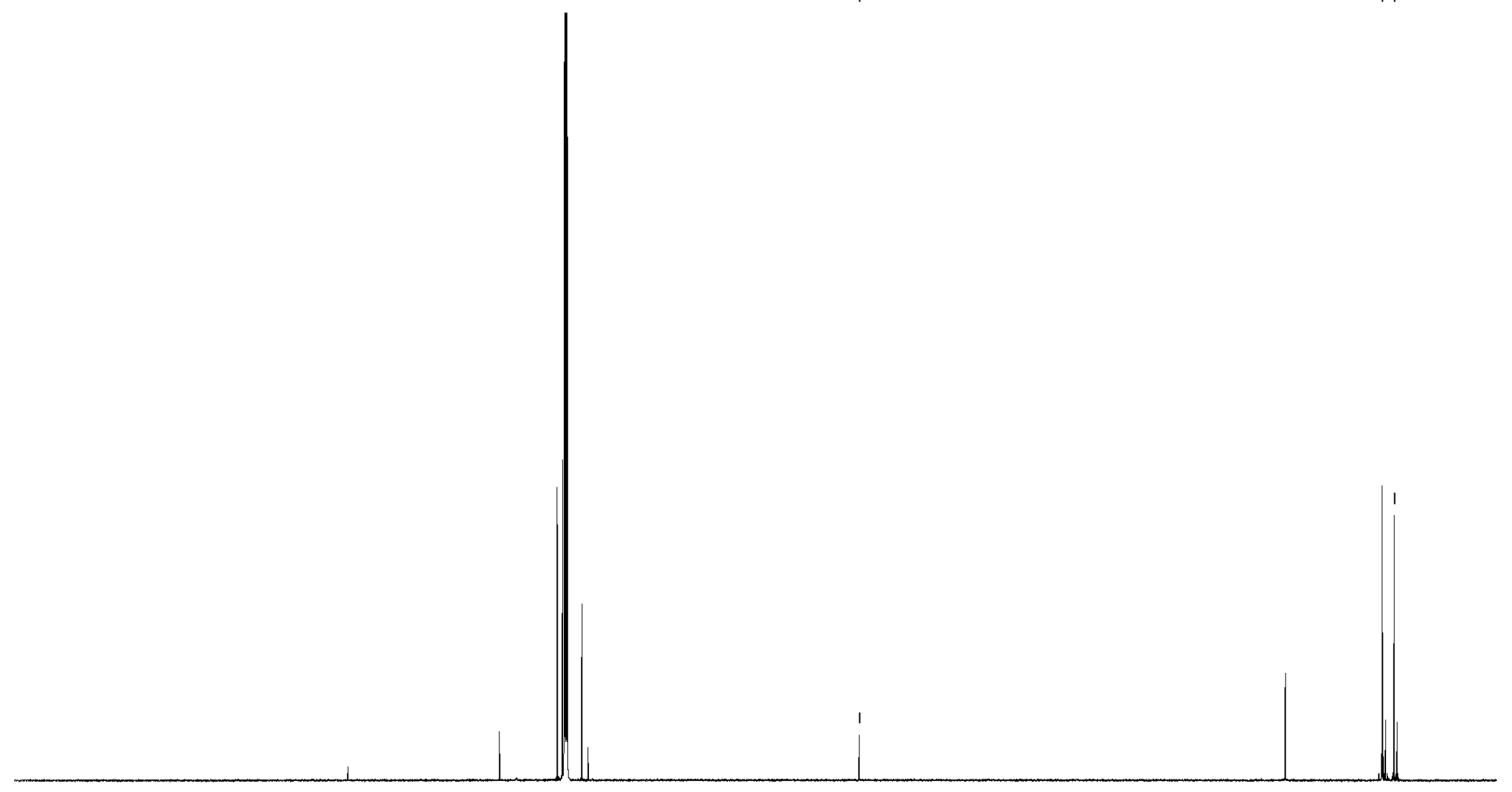


${ }^{1} \mathrm{H}$, Si HMQC NMR (500/99 MHz, $\left.\mathrm{C}_{6} \mathrm{D}_{6}\right)$ :

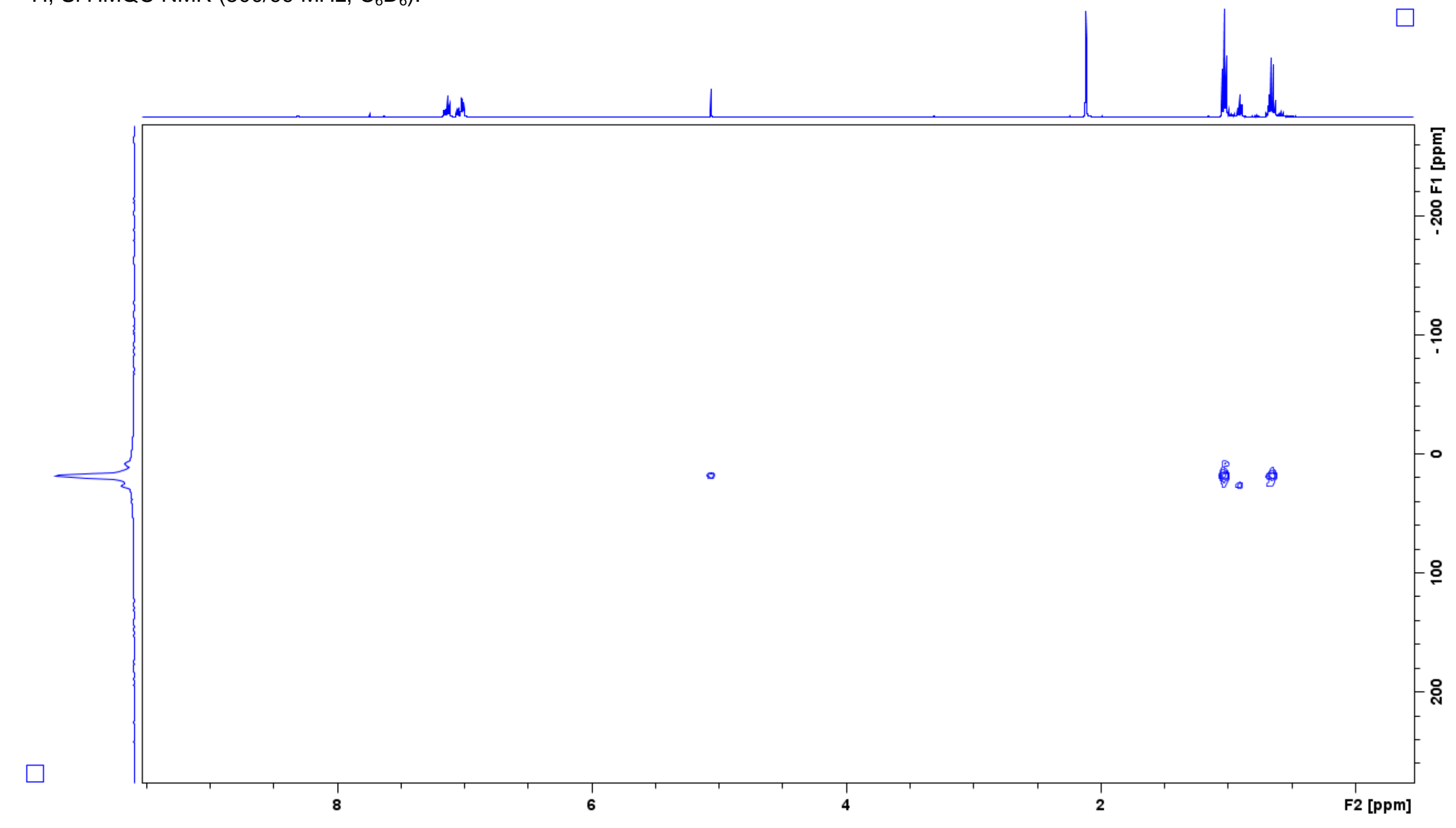


1-Dimethylphenylsilyl-1,4-dihydropyridine (20)

${ }^{1} \mathrm{H}$ NMR $\left(500 \mathrm{MHz}, \mathrm{C}_{6} \mathrm{D}_{6}\right.$ ):

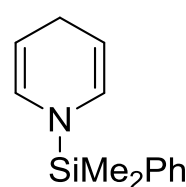

$\mathrm{SiMe}_{2} \mathrm{Ph}$

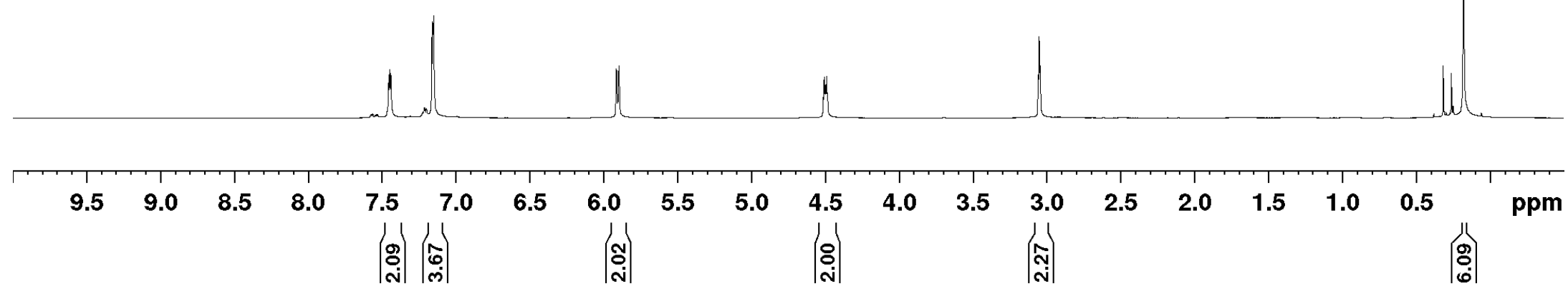


${ }^{13} \mathrm{C}$ NMR $\left(126 \mathrm{MHz}, \mathrm{C}_{6} \mathrm{D}_{6}\right)$ :
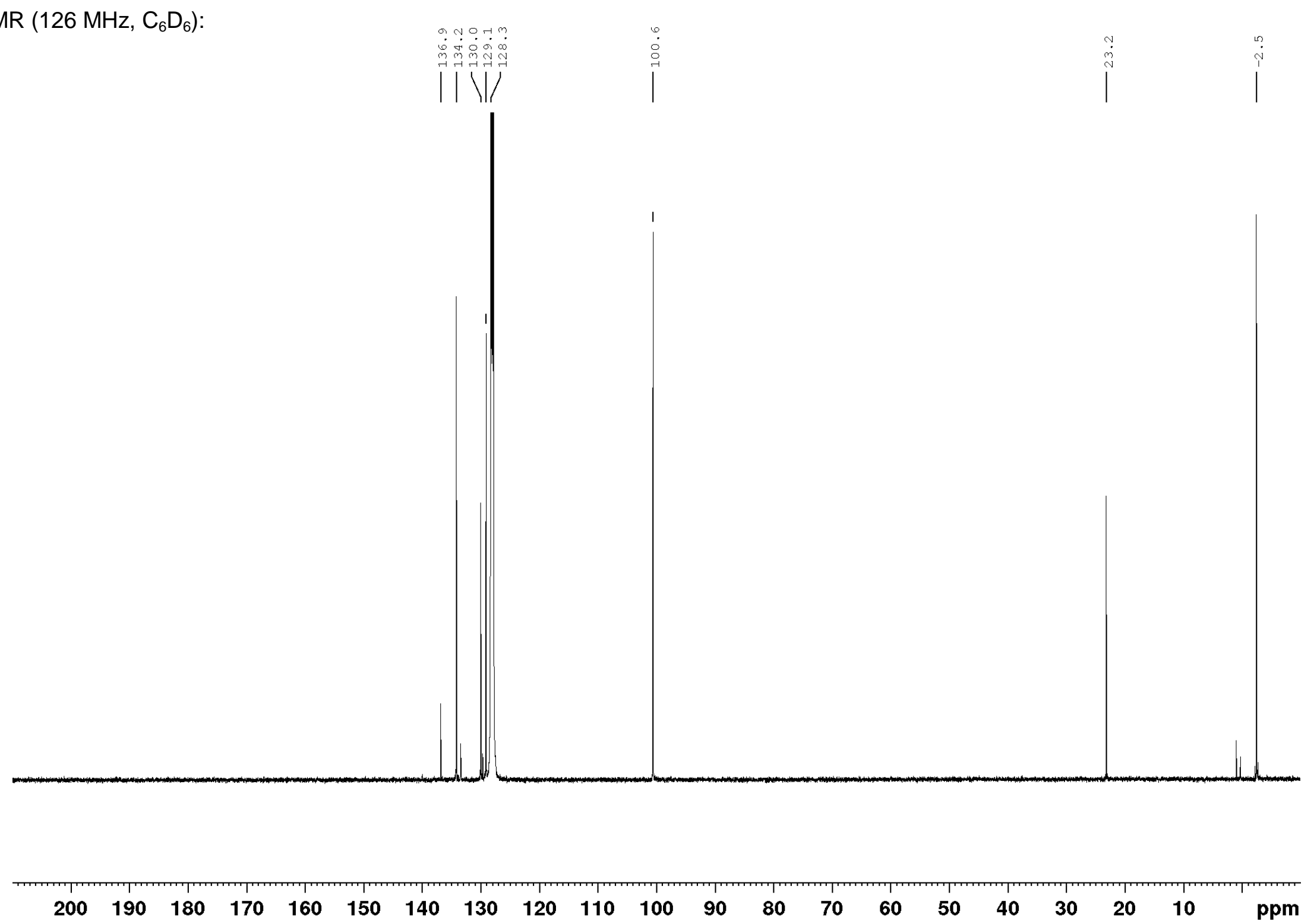
${ }^{29} \mathrm{Si}\left\{{ }^{1} \mathrm{H}\right\}$ DEPT NMR $\left(99 \mathrm{MHz}, \mathrm{C}_{6} \mathrm{D}_{6}\right)$ :

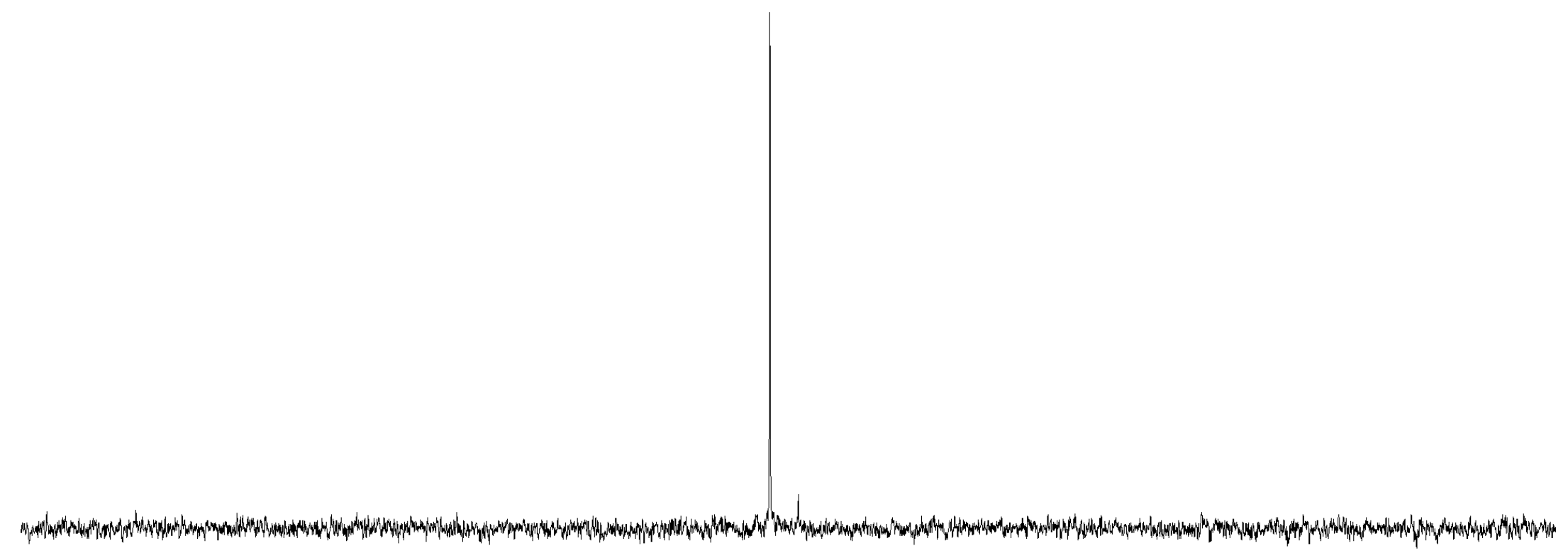

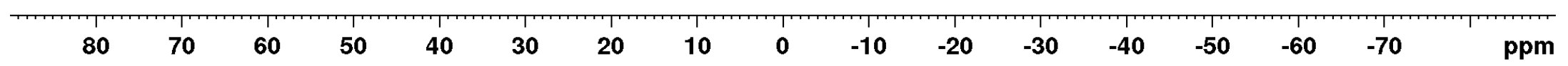




\section{$6 \quad$ References}

[S1] Ansell, M. B.; Roberts, D. E.; Cloke, G. E.; Navarro, O.; Spencer, J., Angew. Chem., Int. Ed. 2015, 54, 5578-5582.

[S2] Prepared according to a modified procedure from: Ohki, Y.; Takikawa, Y.; Sadohara, H.; Kesenheimer, C.; Engendahl, B.; Kapatina, E.; Tatsumi, K., Chem. Asian J. 2008, 3, 1625-1635.

[S3] Prepared according to a modified procedure from: Brookhart, M.; Grant B.; Volpe, Jr., A. F. Organometallics 1992, 11, 3920-3922.

[S4] Omann, L.; Oestreich, M. Angew. Chem., Int. Ed. 2015, 127, 10414-10418.

[S5] Agilent CrysAlis PRO, 2012, Agilent Technologies, Yarnton, UK.

[S6] Sheldrick, G. M., Acta Crystallogr., Sect. A. 1990, 46, 467-473.

[S7] Sheldrick, G. M., Acta Crystallogr., Sect. A. 2008, 64, 112-122.

[S8] Cambridge Crystallographic Data Centre:

http://www.ccdc.cam.ac.uk/Solutions/CSDSystem/Pages/Mercury.aspx.

[S9] Königs, C. D. F.; Klare, H. F. T.; Ohki, Y.; Tatsumi, K.; Oestreich, M. Org. Lett. 2012, $14,2842-2845$.

[S10] Klare, H. F. T.; Oestreich, M.; Ito, J.-i.; Nishiyama, H.; Ohki, Y.; Tatsumi, K. J. Am. Chem. Soc. 2011, 133, 3312-3315.

[S11] (a) Jiang, Y.; Blacque, O.; Fox, T.; Berke, H. J. Am. Chem. Soc. 2013, 135, 77517760. (b) Zheng, J.; Chevance, S.; Darcel, C.; Sortais, J.-B. Chem. Commun. 2013, 49, 10010-10012. (c) Berkefeld, A.; Piers, W. E.; Parvez M. J. Am. Chem. Soc. 2010, 132, 10660-10661. (d) LeBlanc, F. A.; Piers, W. E.; Parvez, M. Angew. Chem., Int. Ed. 2014, 53, 789-792. (e) Metsänen, T. T.; Oestreich, M. Organometallics 2015, 34, 543-546.

[S12] Königs, C. D. F.; Klare, H. F. T.; Oestreich, M. Angew. Chem., Int. Ed. 2013, 52, 10076-10079. 